\title{
Automatic Transition Prediction and Application to High- Lift Multi-Element Configurations
}

\author{
Andreas Krumbein ${ }^{*}$ \\ Deutsches Zentrum für Luft- und Raumfahrt - DLR (German Aerospace Center), Braunschweig, Germany
}

\begin{abstract}
A Reynolds-averaged Navier-Stokes (RANS) solver, a laminar boundary layer code and an $\mathrm{e}^{N}$-database method for transition prediction were coupled in order to perform RANS computations of two-dimensional high-lift multi-element systems with automatic laminarturbulent transition prediction and transitional flow regions. It will be shown, that the coupled system represents a RANS-based CFD tool that provides accurate values of the transition locations during the ongoing RANS computation automatically and fast without the need for the intervention by the code user. Thus, RANS computations of two-dimensional high-lift multi-element configurations with transition can be carried out without $a$ priori knowledge of the transition characteristics of the specific flow problem. The coupling structure and the underlying algorithm of the transition prediction procedure as well as the physical modeling of transitional flow regions and their generation in the RANS computational grid are described. The testing of the transition prediction procedure is described and documented. The computational results are compared to experimental data.
\end{abstract}

\section{Nomenclature}

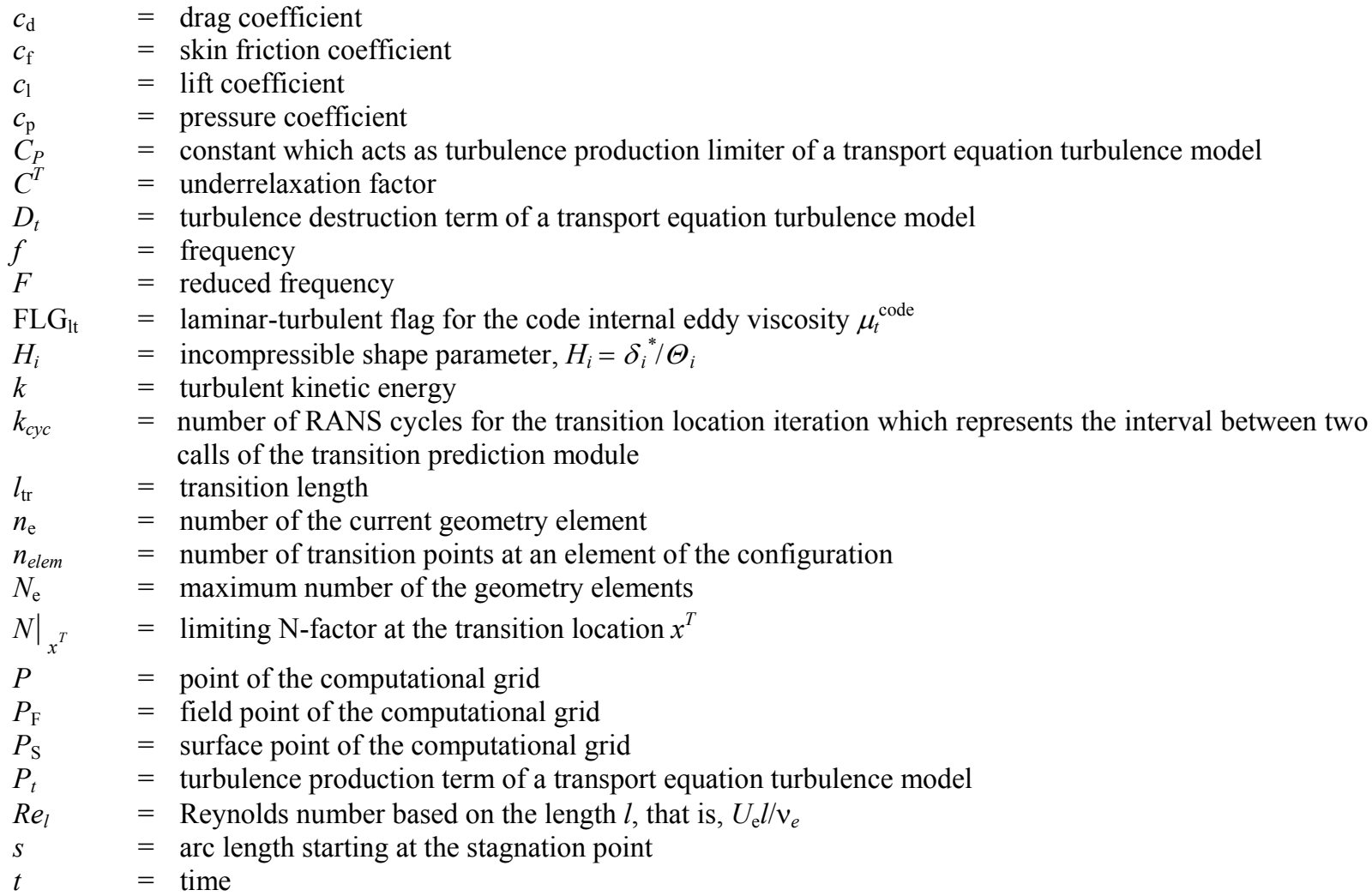

${ }^{*}$ Research Scientist, Design Engineer, Institute of Aerodynamics and Flow Technology, Numerical Methods, Lilienthalplatz 7, D-38108 Braunschweig, andreas.krumbein@dlr.de, AIAA Member. 


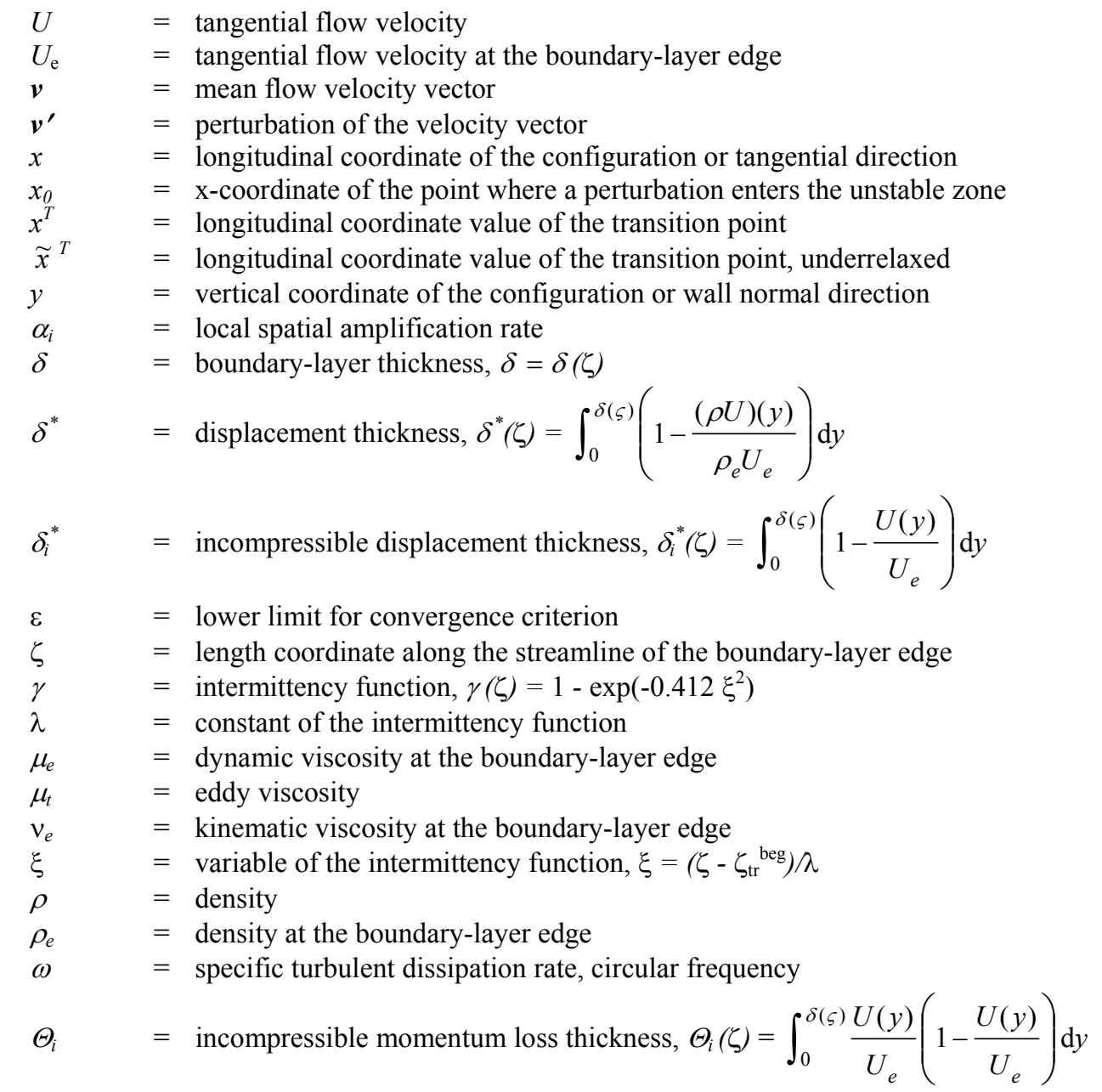

\section{Subscripts}

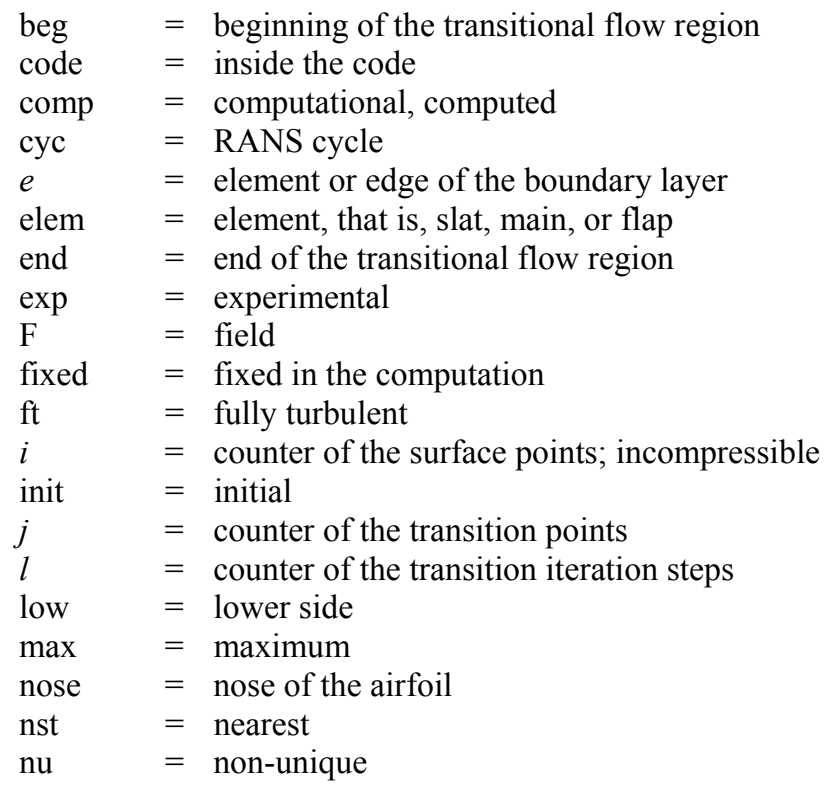




$\begin{array}{ll}\text { old } & =\text { old } \\ q & =\text { placemarker for upper side or lower side } \\ \mathrm{S} & =\text { surface } \\ \text { sep } & =\text { at the separation point } \\ \text { stag } & =\text { at the stagnation point } \\ t & =\text { turbulent } \\ \text { tr } & =\text { at transition onset, transitional region, with transition } \\ \text { trail } & =\text { trailing edge } \\ T & =\text { transition } \\ \text { upp } & =\text { upper side }\end{array}$

\section{Introduction}

$\mathrm{T}$ he modeling of laminar-turbulent transition in Reynolds-averaged Navier-Stokes (RANS) solvers is a necessary requirement for the computation of flows over airfoils and wings in the aerospace industry because it is not possible to obtain quantitatively correct results if the laminar-turbulent transition is not taken into account. For the design process of wings in industry, there exists the demand for a RANS-based computational fluid dynamics (CFD) tool that is able to handle flows automatically and autonomously with laminar-turbulent transition.

The first steps towards the setup of such a tool were made e.g. in Ref. 1, where a RANS solver and an $\mathrm{e}^{N}$-method ${ }^{2,3}$ based on linear stability theory and the parallel flow assumption were applied and in Ref. 4, where a RANS solver, a laminar boundary-layer method ${ }^{5}$, and an $\mathrm{e}^{N}$-method were coupled. There, the boundary-layer method was used to produce highly accurate laminar, viscous layer data to be analyzed by a linear stability code. Hence, the very expensive grid adaptation necessary to produce accurate viscous layer data directly from the NavierStokes grid was avoided. The use of an $\mathrm{e}^{N}$-database method ${ }^{6}$ results in a coupled program system that is able to handle automatically transition prediction. Alternative approaches using a transition closure model or a transition/turbulence model directly incorporated into the RANS solver are documented in Refs. 7-9.

At the Deutsches Zentrum für Luft- und Raumfahrt, German Aerospace Center (DLR), the block structured RANS code FLOWer ${ }^{10}$ is used together with the laminar boundary-layer method in Ref. 5 and the $\mathrm{e}^{N}$-database method in Ref. 6. The laminar boundary-layer method and the $\mathrm{e}^{N}$-database method form a so called 'transition prediction module' that is coupled to the RANS solver and that interacts with the RANS solver during the computation. ${ }^{11,12}$ Presently, the transition prediction module of FLOWer can be applied to two-dimensional oneelement or multi-element configurations.

The description of transitional flow regions in FLOWer is done either by the application of point transition or by the application of physical models of transitional flow. The application of point transition means that turbulence quantities, which are suppressed in the laminar part of the flow, suddenly become active at the location of transition onset. This procedure results in a sudden change of the flow quantities in this area. Due to the effects of numerical dissipation a small transitionallike flow region is generated artificially in a computation without physical transition modeling. However, the sudden change of the flow quantities is often strong enough to prevent the convergence of the iterative transition prediction process. ${ }^{13}$ In addition, the application of point transition generates a strong upstream influence so that the transitionallike flow region starts considerably upstream of the transition location. In two-dimensional airfoil flows, an upstream influence up to $10 \%$ of the chord length of the airfoil can be observed. The physical modeling of transitional flow overcomes this problem and leads to a more stable coupled computational procedure of RANS code and transition prediction module on the one hand and to better results of the numerical simulation on the other.

In Ref. 14 it was described how the FLOWer code was extended to handle multi-element configurations with transition. The extensions were performed in two steps. First, a generalized infrastructure in the FLOWer code with respect to the transition prediction module was built up and tested, that is, the code was changed in such a way that the transition prediction module can be activated now for arbitrary multi-element configurations independent of the block topology and the grid structure. Second, the transition prediction module was coupled to the generalized infrastructure. In the framework of Ref. 14, the first extension step was documented. Thus, Ref. 14 has the character of a progress report. In the present paper, the second extension step is described and finalizes the documentation of the treatment of automatically predicted transition locations and their application in a RANS code with physically modeled transitional flow regions for two-dimensional multi-element configurations. At the time that Ref. 14 was written, the transition prediction module was not yet coupled to the extended FLOWer code; the locations of laminar separation determined by the FLOWer code were supposed to represent the laminar-turbulent transition locations in a first step. In many cases, this assumption leads to a good approximation of the real transition point, particularly for 
low Reynolds number airfoil flows, when transition does not occur before the laminar boundary layer separates. Now the transition locations are determined by the $\mathrm{e}^{N}$-database method of Ref. 6 .

The main objective of the work presented in this paper is to supply a reliable infrastructure in a RANS code and a coupling structure between the RANS code and the transition prediction module so that both program systems can be used for the computation of two-dimensional multi-element high-lift systems of aircraft including transitional flow regions and automatically predicted transition locations. In the end, it will be shown that the coupled system consisting of the FLOWer code and the transition prediction module represents a RANS-based CFD tool that provides accurate values of the transition locations during the ongoing RANS computation automatically and fast without the need for the intervention of the user.

A method and an algorithm for the coupling of the transition prediction module and thereby for detecting the laminar-turbulent transition locations based on linear stability theory were implemented in the FLOWer code. For each element of a high-lift configuration on the upper and lower side, the laminar separation point will be detected, and the transition fixed there during the transient phase of the RANS computation. When the computation has reached a stable state and the lift coefficient has reached a certain convergence level, the transition point will be detected by the transition prediction module and the transition will be fixed there as long as a new laminar separation point or a new transition point is detected. The intermittency function and three transition length models were implemented and validated in a variety of test computations for two selected high-lift multi-element test cases. This paper focuses on the structure of the transition prediction module and its coupling to the FLOWer code, the underlying algorithm of the transition prediction procedure $e^{4-6,11,12}$ and the testing of the algorithm. The testing is described and documented by a number of commented plots of the results of the transition prediction procedure and of the transition length models. The computed pressure and skin friction distributions are presented and the $c_{\mathrm{p}}$-distributions are compared to experimental data. Finally, the global lift and drag coefficients are compared with experimental findings.

\section{Implementation}

FLOWer is a three-dimensional, compressible RANS code for steady or unsteady flow problems and uses structured body-fitted multiblock meshes. The code is based on a finite volume method and a cell-vertex spatial discretization scheme and uses an explicit Runge-Kutta time integration scheme with multigrid acceleration. The influence of turbulence is taken into account by eddy viscosity turbulence models according to the Boussinesq approximation. The transition handling is independent of the block topology of the computational grid and of the grid structure (structured, unstructured or hybrid grid). ${ }^{11}$

\section{A. Coupling of the RANS solver and the transition prediction module}

The complete coupled program system that is used for transition prediction with the RANS solver FLOWer consists of the RANS solver itself, ${ }^{10}$ a laminar boundary-layer method for swept, tapered wings ${ }^{5}$ and a transition prediction method, which is provided with all necessary data, for example, boundary-layer parameters, by the laminar boundary-layer method. Besides a number of empirical transition criteria, the most general transition prediction method that is available in the FLOWer transition prediction module is an $\mathrm{e}^{N}$-database method. ${ }^{6}$

The RANS solver communicates the surface pressure distribution of the configuration as input data to the laminar boundary-layer method, the laminar boundary-layer method computes all of the boundary-layer parameters that are needed for the transition prediction method and the transition prediction method determines new transition locations that are given back to the RANS solver. This coupled structure results in an iterative procedure for the transition locations within the iteration of the RANS equations.

After a certain number of iteration cycles, $k_{c y c}$, of the RANS solver, the transition prediction module is called. With the call of the module the solution process is interrupted. The module analyzes the laminar boundary layers of specified components of the configuration, for example, one

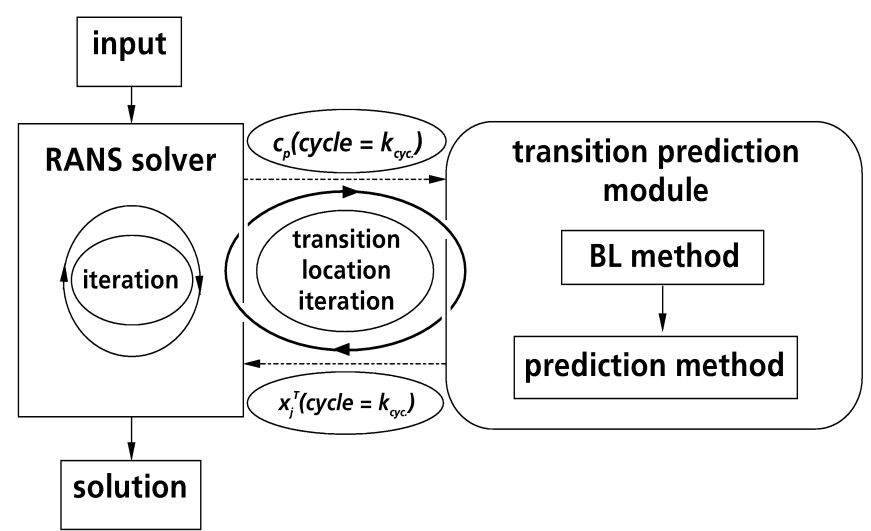

Fig. 1 Coupling structure of the RANS solver and the transition prediction module. 
element of a two-dimensional airfoil configuration. The determined transition locations, $x_{j}^{T}\left(c y c l e=k_{c y c}\right)$ with $j=1, \ldots, n_{\text {elem }}$, where $n_{\text {elem }}$ is the number of transition points, are communicated back to the RANS solver, which performs transition prescription by applying an transition setting algorithm, ${ }^{11,12}$ and continues the solution process of the RANS equations. In so doing, the determination of the transition locations becomes an iteration process itself. The structure of the approach is outlined graphically in Fig. 1. At every call of the module the surface pressure, $c_{\mathrm{p}}\left(\right.$ cycle $=k_{\text {cyc }}$ ), along an airfoil element computed by the RANS solver is used as input to the boundary-layer calculation. The viscous data calculated by the boundary-layer method is subsequently analyzed by the $\mathrm{e}^{N}$-database method. The application of a boundary-layer method for the computation of all viscous data necessary for the transition prediction method ensures the high accuracy of the viscous data required by the $\mathrm{e}^{N}$-methods for the analysis of laminar boundary layers. Thus, as shown in Ref. 4, the large number of grid points near the wall for a high resolution of boundary layers, the adaptation of the Navier-Stokes grid in the laminar and turbulent boundarylayer regions and the generation of new adapted grids for the RANS solver after every step of the transition location iteration are avoided and the computational time can be massively reduced. The algorithm for the transition prediction iteration works as follows:

1) The RANS solver is started as if a computation with prescribed transition locations should be performed. In this moment, the transition locations are set far downstream on the upper and lower sides of the airfoil, for example, at the trailing edge. The RANS solver now computes a fully laminar flow over the airfoil.

2) During the solution process of the RANS equations the laminar flow is checked for laminar separation points by the RANS solver. In the case that a laminar separation is detected, the separation point is used as an approximation of the transition location, the transition is fixed there and the computation is continued.

3) The RANS equations are iterated until the lift coefficient $c_{l}=c_{l}$ (cycles) has become constant with respect to the iteration cycles.

4) The transition prediction module is called. The laminar boundary-layer method determines the locations of laminar separation on upper and lower sides of the airfoil. The $\mathrm{e}^{N}$-database method determines the transition locations on upper and lower sides of the airfoil. In the case that the $\mathrm{e}^{N}$-database method does not detect a transition location due to Tollmien-Schlichting instabilities upstream of the current laminar separation point from the boundary-layer method, the laminar separation point is used as an approximation of the real transition point and transition is fixed there.

5) The current coordinate $x_{j}^{T}\left(\right.$ cycle $\left.=k_{c y c}\right)$, which is used as a transition location, is underrelaxed. That is, as new transition locations the coordinates $\tilde{x}_{j}^{T}\left(\right.$ cycle $\left.=k_{c y c}\right)$, which are located downstream of the coordinates $x_{j}^{T}\left(\right.$ cycle $\left.=k_{c y c}\right)$, are used,

$$
\tilde{x}_{j}^{T}\left(k_{c y c}\right)=C_{j}^{T}\left(k_{c y c}\right) x_{j}^{T}\left(k_{c y c}\right) \quad \text { with } \quad j=1, \ldots, n_{\text {elem }},
$$

with $C_{j}^{T}\left(k_{c y c}\right)>1$. Only after the last step of the transition location iteration $C_{j}^{T}\left(k_{c y c}\right)=1$ is applied. This underrelaxation of the transition locations prevents the case that at an unconverged stage during the transition location iteration, transition coordinates are determined too far upstream, and might not be shifted downstream again.

6) As a convergence criterion $\Delta \widetilde{x}_{j}^{T, l}<\varepsilon$ with $\Delta \widetilde{x}_{j}^{T, l}=\left|\widetilde{x}_{j}^{T}\left(k_{c y c}^{l}\right)-\tilde{x}_{j}^{T}\left(k^{l-1}{ }_{c y c}\right)\right|$ is applied, where $l$ is the current iteration step. In the case that the criterion is satisfied, the iteration for $x_{j}^{T}$ is finished, else the algorithm loops back to station 2).

\section{B. $\mathrm{e}^{N}$-Database Method}

The $\mathrm{e}^{N}$-method used ${ }^{5}$ applies

$$
\left.N\right|_{x}{ }^{T}=-\int_{x_{0}}^{x^{T}} \alpha_{i} d x
$$

as a transition criterion. The limiting $\mathrm{N}$-factor $\left.N\right|_{x^{T}}$ at the transition location $x^{T}$ must be determined experimentally and represents the total amplification of a perturbation of the mean flow of frequency $f$ at the transition location. $x_{0}$ is the $\mathrm{x}$-coordinate of the point where this perturbation enters the unstable zone, and $\alpha_{i}$ is the local spatial amplification rate. The perturbation of the mean flow $v$ of frequency $f-\boldsymbol{v}$ is assumed to be steady and well parallel - is described by a harmonic Tollmien-Schlichting wave 


$$
\boldsymbol{v}^{\prime}=\boldsymbol{v}_{0}^{\prime}(y) e^{i(\alpha x-\omega t)}
$$

with the circular frequency $\omega=2 \pi f \delta_{i}^{*} / U_{e}$ and the incompressible displacement thickness $\delta_{i}^{*}=\int_{0}^{\delta}\left(1-\frac{U(y)}{U_{e}}\right) \mathrm{d} y$, and $\alpha=\alpha_{r}+i \alpha_{i} . \alpha_{i}$ can be expressed as a function of the shape parameter $H_{i}, R_{\delta_{i}^{*}}$, the Reynolds number with respect to the incompressible displacement thickness $\delta_{i}^{*}$, and the reduced frequency $F$,

$$
\alpha_{i}=\alpha_{i}\left(H_{i}, \operatorname{Re}_{\delta_{i}^{*}}, F\right)
$$

where

$$
\begin{gathered}
H_{i}=\delta_{i}^{*} / \Theta_{i} \text { with } \Theta_{i}=\int_{0}^{\delta} \frac{U(y)}{U_{e}}\left(1-\frac{U(y)}{U_{e}}\right) \mathrm{d} y, \\
\operatorname{Re}_{\delta_{i}^{*}}=\frac{U_{e} \rho_{e} \delta_{i}^{*}}{\mu_{e}}, \\
F=\frac{2 \pi f \mu_{e}}{\delta_{e} U_{e}^{2}}=\frac{\omega}{\operatorname{Re}_{\delta_{i}^{*}}} .
\end{gathered}
$$

As described in Refs. 5 and 17, the boundary layers of the similarity solution using the approach of Falkner and Skan were generated for 13 shape parameters $H_{i}$, which cover the range from highly accelerated up to separating flows. The growth of the boundary layer was simulated by varying $R e_{\delta_{i}^{*}}$. For each boundary layer, stability computations were completed for a sufficient large range of excited frequencies. The results for the amplification rates $\alpha_{i}$ were stored in a database. The stability computation for a real boundary layer, using the database method, is executed for a given frequency $f$ in $\mathrm{Hz}$ in the following way: At each grid point on the airfoil the properties $f, R_{\delta_{i}^{*}}$, $H_{i}, U_{e}, \mu_{e}$ and $\rho_{e}$ are known. Evaluating $F$ from the value of $f, \alpha_{i}$ is obtained from $\alpha_{i}=\alpha_{i}\left(H_{i}, \operatorname{Re}_{\delta_{i}^{*}}, F\right)$ via interpolation in the database.

In Refs. 5 and 17, it was shown that the use of the database is a high quality approximation for the values resulting from a local linear stability code. In Refs. 4, 5, 17 and 18, it was shown that the Falkner-Skan approach approximates all relevant properties of the two-dimensional laminar boundary-layer flow around airfoils in practice, with high accuracy.

For the application of the $\mathrm{e}^{N}$-method extensive experimental programs have been performed to determine the value of $\left.N\right|_{x}{ }^{T}$ which depends on the environmental disturbances in the flow. For free flight conditions $\left.N\right|_{x} \approx 12$ was found for Tollmien-Schlichting waves. For wind tunnel flows, which are normally characterised by relatively high disturbances, one finds $\left.N\right|_{x} \approx 6$.

\section{Generation of Transitional Flow Regions}

In the case that a new transition location has been determined, the laminar, transitional and turbulent flow regions must be generated anew within the computational grid. The generation of the different regions is done by the setting of a real value flag $\mathrm{FLG}_{\mathrm{lt}}$ at each point of the computational grid. $\mathrm{FLG}_{\mathrm{lt}}$ is applied to the value of the turbulence production $P_{t}$ of a transport equation turbulence model, which is computed for every point $P$ in the flow field. $\mathrm{FLG}_{\mathrm{lt}}$ is applied in the following way: 


$$
P_{t}^{\text {code }}(P)=\min \left(P_{t}(P), D_{t}(P) C_{P}{ }^{\mathrm{FLG}_{\mathrm{lt}}(P)}\right)
$$

with $\mathrm{FLG}_{\mathrm{lt}}(P)=0.0$ for a laminar grid point, $\mathrm{FLG}_{\mathrm{lt}}(P)=1.0$ for a turbulent grid point and $\mathrm{FLG}_{\mathrm{lt}}(P)=\gamma(P)$ for a transitional grid point, $\gamma(P)$ being the value of the intermittency function $\gamma$ at the grid point $P$. $D_{t}$ is the value of the turbulence destruction of a transport equation turbulence model and $C_{P}$ is a constant which acts as a limiter on the turbulence production term for the source term of the turbulence model. ${ }^{10-11}$ Thus, the source term of the turbulence model is controled in such a way that its value is lower or equal zero in laminar regions. The default value for $C_{P}$ in the FLOWer code is $C_{P}=10$ for 2-equation turbulence models of k- $\omega$ type and $C_{P}=10^{10}$ for 1-equation turbulence models. ${ }^{10}$ The default value of $C_{P}$ for 1-equation turbulence models in effect turns off the production limitation in fully turbulent regions for these cases. In the computations which were performed for the present work the default values for $C_{P}$ were used. The assignment of the values of $\mathrm{FLG}_{\mathrm{It}}$ is done first for the grid points on solid walls of the configuration.

The laminar length on the upper or lower sides of an airfoil is defined by the interval between the stagnation point and the transition point on the side $q$, with $q=$ upp, low indicating either the upper or the lower side of the airfoil, $0 \leq s_{q} \leq s_{q, \mathrm{tr}}$ beg, where $s_{q}$ is the arc length on the side $q$ starting at the stagnation point. The turbulent length is defined by the interval between the ending point of the transitional region and the trailing-edge point on side $q, s_{q, \mathrm{tr}}^{\text {end }} \leq s_{q} \leq s_{q}^{\text {trail }}$, and the transitional length is the interval between the transition point and the ending point of the transitional region, $s_{q, \mathrm{rr}}^{\text {beg }} \leq s_{q} \leq s_{q, \mathrm{tr}}$ end . The different intervals are depicted in Fig. 2.

Here $\gamma$ is expressed as

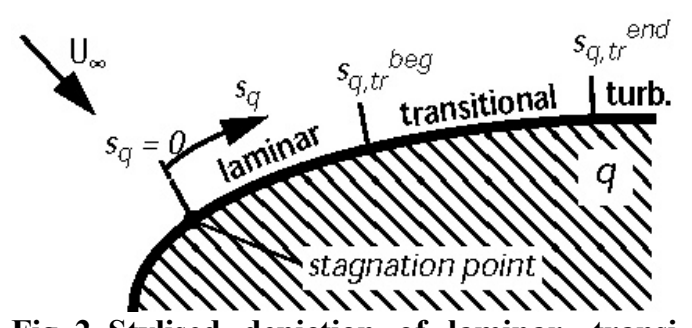

Fig. 2 Stylised depiction of laminar, transitional and turbulent flow regions at surface side $q$.

$$
\gamma(x)=1-\exp \left(-0.412 \xi^{2}\right)
$$

with

$$
\xi=\left(x-x_{\mathrm{tr}}^{\mathrm{beg}}\right) / \lambda
$$

according to Ref. 15, where $x$ is the longitudinal coordinate of a flat plate with its origin located at the upstream end of the plate and $\lambda$ being a measure of the extent of the transitional region. According to Ref. 13, the ending point of the transitional region $x_{\mathrm{tr}}{ }^{\text {end }}$ can be defined as

$$
x_{\mathrm{tr}}^{\mathrm{end}}=x(\gamma=0.99)
$$

which yields

$$
\lambda=\left(x_{\mathrm{tr}}^{\mathrm{end}}-x_{\mathrm{tr}}^{\mathrm{beg}}\right) / 3.36 .
$$

For the determination of the extent of the transitional region, the transition length $l_{\mathrm{tr}}=x_{\mathrm{tr}}{ }^{\text {end }}-x_{\mathrm{tr}}^{\text {beg }}$, the formulas from Ref. 16,

$$
R e_{l_{t r}}=5.2\left(R e_{x_{t r t}^{\text {beg }}}\right)^{\frac{3}{4}}
$$

for flows without pressure gradient, and

$$
R e_{l_{t r}}=2.3\left(R e_{\delta_{t r}^{*} \text { tog }}\right)^{\frac{3}{2}}
$$


for flows with pressure gradient, are applied, as is recommended in Ref. 13 for flows in which transition does not occur before laminar separation. Whereas Eq. (13) is applied, when a laminar transition point is determined by the RANS code during the transient phase of the computation, Eq. (14) is used, when the transition prediction module has analyzed the laminar boundary layers and the $\mathrm{e}^{N}$-database method has not found a transition point due to Tollmien-Schlichting instabilities upstream of the current laminar separation point, determined by the laminar boundary-layer method. In this case, the laminar separation point from the laminar boundary-layer code is used as an approximation of the real transition point and transition is fixed there. In all computational tests, the laminar separation point determined by the laminar boundary-layer method has always been located upstream of the laminar separation point determined by the RANS code, and the computational results using the laminar separation point from the boundary-layer method compared slightly better with experimental values than those using the laminar separation point from the RANS code.

For a predicted transition point which is determined by the $\mathrm{e}^{N}$-database method the formula

$$
R e_{l_{t r}}=4.6\left(R e_{\delta_{\text {tr }}^{* \text { beg }}}\right)^{\frac{3}{2}}
$$

according to Ref. 16 is applied. Here and in Eq. (14)

$$
\delta^{*}(x)=\int_{0}^{\delta(x)}\left(1-\frac{(\rho U)(y)}{\rho_{e} U_{e}}\right) \mathrm{d} y .
$$

The thickness of the laminar boundary layer $\delta$ is evaluated according to a procedure described in Ref. 4. Thus $\delta^{*}$ and $\rho_{e}$ and $U_{\mathrm{e}}$, the values of the density and the tangential flow velocity at the boundary layer edge, can be determined.

For the implementation in the RANS solver, the $x$-coordinate in the formulas is replaced by the arc length $s$. Here $s_{q, \mathrm{tr}}{ }^{\mathrm{beg}}$ is given either by the location of a laminar separation point, for formula (a) or formula (b), or by a predicted transition point, for formula (c), and $s_{q, \text { tr }}$ end is determined by formula (a), based on Eq. (14), formula (b), based on Eq. (13), or formula (c), based on Eq. (15), respectively. Formula (a) reads

$$
s_{q, \text { tr }}^{\text {end(a) }}=2.3 \sqrt{\left(U_{e} / v_{e}\right)_{s_{q, \mathrm{tr}}^{\mathrm{beg}}}}\left(\delta_{s_{q, \mathrm{tr}}^{\mathrm{beg}}}^{*}\right)^{\frac{3}{2}}+s_{q, \mathrm{tr}}^{\text {beg }} .
$$

Formula (b) reads

$$
s_{q, \mathrm{tr}}^{\text {end(b) }}=5.2\left[\left(U_{e} / v_{e}\right)_{s_{q, \mathrm{tr}}^{\mathrm{beg}}}\right]^{-\frac{1}{4}}\left(s_{q, \mathrm{tr}}^{\mathrm{beg}}\right)^{\frac{3}{4}}+s_{q, \mathrm{tr}}^{\mathrm{beg}} .
$$

Formula (c) reads

$$
s_{q, \mathrm{tr}}^{\mathrm{end}(\mathrm{c})}=4.6 \sqrt{\left(U_{e} / v_{e}\right)_{s_{q, \mathrm{tr}}^{\mathrm{beg}}}}\left(\delta_{s_{q, \mathrm{br}}^{\mathrm{beg}}}^{*}\right)^{\frac{3}{2}}+s_{q, \mathrm{tr}}^{\mathrm{beg}},
$$

and the intermittency function $\gamma$ is applied in the form

$$
\gamma\left(s_{q}\right)=1-\exp \left[-0.412\left(3.36 \frac{s_{q}-s_{q, \mathrm{tr}}^{\text {beg }}}{s_{q, \mathrm{tr}}^{\text {end }}-s_{q, \mathrm{tr}}^{\mathrm{beg}}}\right)^{2}\right] .
$$

After all of the surface points on upper and lower side of an airfoil have been assigned to either the corresponding laminar, turbulent or transitional interval, the field points, all points apart from the solid walls, are 
treated in the following way. ${ }^{11}$ Within a limiting wall normal distance that can be adjusted by the user of the code, every field point $P_{\mathrm{F}}$ assumes the flag value of the surface point $P_{\mathrm{S}}{ }^{\text {nst }}$ that is located nearest to $P_{\mathrm{F}}$,

$$
\mathrm{FLG}_{\mathrm{lt}}\left(P_{\mathrm{F}}\right)=\mathrm{FLG}_{\mathrm{lt}}\left[P_{\mathrm{S}}{ }^{\text {nst }}\left(P_{\mathrm{F}}\right)\right] \text {. }
$$

By this treatment, a laminar and a transitional zone for the current element are generated within the turbulent remainder of the computational grid. A partitioning into pure laminar zones within the turbulent remainder of the flow domain (point transition) is shown in Fig. 3. All the steps of this procedure must be applied to all elements $n_{\mathrm{e}}, 1 \leq n_{\mathrm{e}} \leq N_{\mathrm{e}}$ of the configuration for which transition prediction is performed. The order of the elements within the procedure is irrelevant.
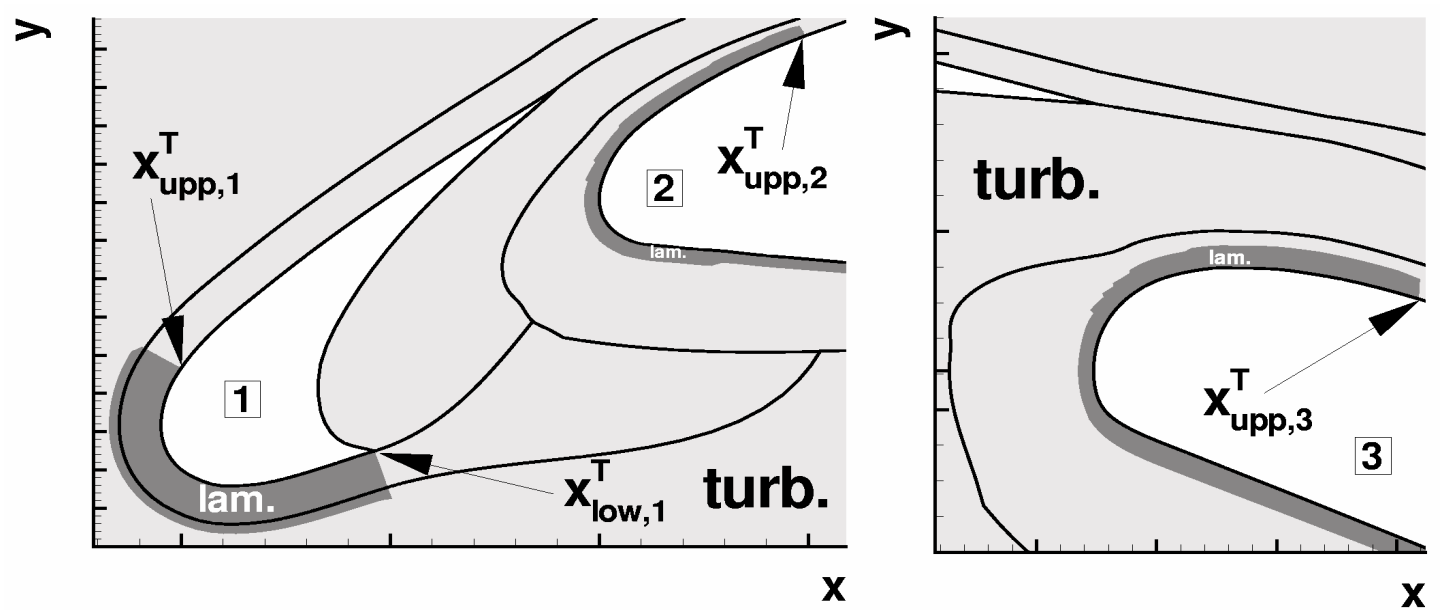

Fig. 3 Laminar zones of a two-dimensional three-element airfoil configuration in a turbulent remainder of the flow domain, point transition, slat and main airfoil (left), flap (right).

\section{Computations}

\section{A. Test Cases}

The test cases used to investigate the automated transition prediction functionality of the coupled system are the two-dimensional three-element take-off and landing configurations of a representative civil aircraft consisting of slat, main airfoil and flap. ${ }^{19-22}$ The structured computational grid ${ }^{21}$ consists of about 80,000 grid points in 9 blocks. The configuration has sharp trailing edges on the lower sides of the slat and the main airfoil, and blunt trailing edges on the slat and main airfoil upper sides and at the end of the flap. The turbulence models used are the SpalartAllmaras one-equation mode ${ }^{23}$ with Edwards and Chandra modification ${ }^{24}$ (SAE) for the take-off case, and the standard Wilcox $\mathrm{k}-\omega$ two-equation model $\mathrm{l}^{25}$ for the landing case. The turbulence models used were chosen according to Ref. 26. As shown in Refs. 11 and 27, the values of the predicted transition locations are almost not influenced by the turbulence model used in the RANS solver when the present transition prediction approach - RANS solver, laminar boundary layer code and $\mathrm{e}^{N}$ transition prediction - is applied. Thus, the use of different turbulence models for the validation of the applied transition prediction procedure is supposed to be justified.

The aerodynamic parameters used in the computations are $\mathrm{M}_{\infty}=0.22, \alpha=21.4^{\circ}, \mathrm{Re}_{\infty}=6 \times 10^{6}=\mathrm{Re}^{\mathrm{lo}}$ and $\operatorname{Re}_{\infty}=16.8 \times 10^{6}=\mathrm{Re}^{\text {hi }}$ for both configurations. For the $\mathrm{e}^{N}$-database method ${ }^{6}$ the value of the limiting $\mathrm{N}$-factor $\left.N\right|_{x^{T}}$ was set to $\left.N\right|_{x^{T}}=9$ according to Ref. 28 . All computations were started with initially set transition locations at $95 \%$ of the particular element's chord length, $x_{\mathrm{tr}, \text { elem }}$ init $/ \mathrm{c}_{\mathrm{elem}}=0.95$ with elem $=$ slat, main or flap, on upper and lower sides. In order to prevent difficulties which may arise due to a movement of the stagnation point in the transient phase of the computation ${ }^{14}$, all computations were initialized with solutions of a certain convergence level from computations with fixed transition locations where the values of the upper side transition points have been reasonably guessed, $x_{\text {tr,elem }}$ fixed $/ \mathrm{c}_{\text {elem }} \approx 0.2$ for the take-off case and $x_{\text {tr,elem }}$ fixed $/ \mathrm{c}_{\text {elem }} \approx 0.1$ for the landing case. In the transition prediction iteration, the RANS cycle interval between two consecutive calls of the transition prediction 
module was set to $\Delta k_{c y c}=2,000$, and for the RANS computations a three-level multigrid method was applied. The experimental data is taken from Ref. 22.

\section{B. Results}

The computations of all cases result in a flow structure that is typical for an aircraft multi-element high-lift configuration before maximum lift. The flow is fully attached on the lower sides of the slat, the main airfoil and the flap. In the coves of the main airfoil and of the slat, separation bubbles are located. The separation bubble in the slat cove is significantly smaller in the computations with transition than in fully turbulent computations. The $c_{\mathrm{p}}$-distribution on the slat upper side is characterized by a high suction peak in all cases. On the upper sides of the main airfoil and the flap, the $c_{\mathrm{p}}$-distributions show spiky perturbations coming from the contour kinks where the trailing edges of the slat and the main airfoil are located when the configuration is undeflected. In all cases, on the lower sides of the slat, the main airfoil and the flap the flow remains fully laminar from the stagnation point up to the intitially set lower transition points at $95 \%$.

The $\mathrm{Re}^{\mathrm{lo}}$-take-off case exhibits a stationary separation bubble on the rear $14 \%$ of the main airfoil upper side, in contrast to the $\mathrm{Re}^{\text {hi }}$-take-off case where the flow is fully attached. For both cases the flow on the flap and slat upper sides is fully attached. For both Reynolds numbers, the flow over the landing configuration is characterized by trailing edge separation bubbles on the upper sides of the slat and the flap. The extent of the slat separation bubble is about $4 \%$ of the slat chord length in the $\mathrm{Re}^{\mathrm{lo}}$-landing case and about $10 \%$ in the Re ${ }^{\text {hi }}$-landing case. For both Reynolds numbers, the extent of the flap separation bubble is about $16 \%$ of the flap chord length. A monitoring of the separation points shows that for both Reynolds numbers the slat separation point and for the $\mathrm{Re}^{\mathrm{lo}}$-landing case also the flap separation point oscillates very slightly so that the convergence of the computations which were performed using local time stepping in order to compute steady solutions is visibly affected. The density residual leveled out at an average value of about $10^{-2}$. The convergence histories of the density residual, the lift coefficient $c_{l}$ and the drag coefficient $c_{d}$ are oscillating around steady average

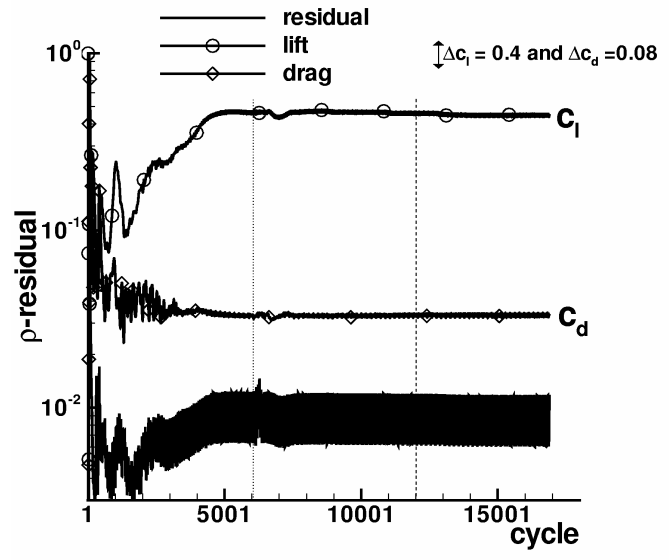

Fig. 4 Convergence history of the RANS computation, low Reynolds number landing case, dotted: transition prediction start, dashed: transition prediction end. values. For $c_{l}$ and $c_{d}$, the corresponding steady average values are supposed to represent the steady state values. In Fig. 4 this behavior is depicted for the $\mathrm{Re}^{\mathrm{lo}}$-landing case. The dotted line at cycle $\approx 6,000$ marks the situation directly after the restart using the restart solution with fixed transition locations; here the transition prediction procedure with

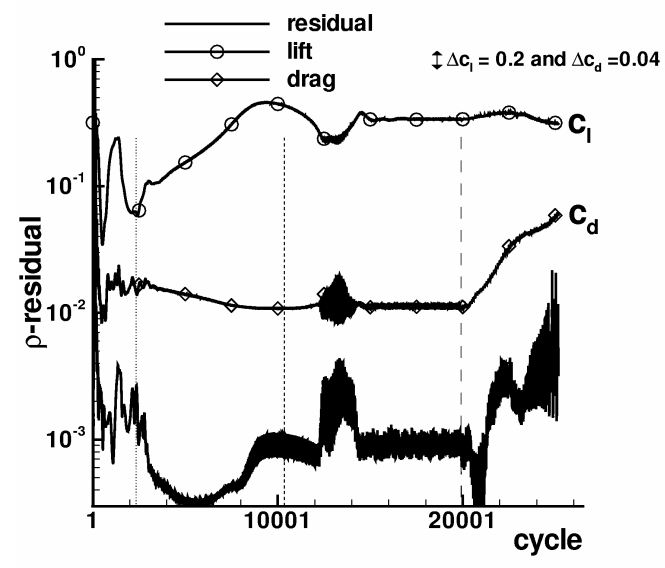

Fig. 5 Convergence history of the RANS computation, low Reynolds number take-off case, dotted: transition prediction start, dashed: transition prediction end, long dashed: start of unsteady computation. initial transition locations which were set almost at the trailing edges starts. The final step of the transition location iteration is done at the cycle marked with a dashed line. Apart from the oscillations in the curves of the force coefficients, the convergence histories are very smooth and after the first part of the transient phase where perturbations are well damped all evolution tendencies are clear. The behavior of the $\mathrm{Re}^{\mathrm{hi}}$-landing case is very similar.

For the take-off cases, the convergence stage of the restart solutions were of very different character. After about 2,350 RANS cycles, the convergence stage of the computations with fixed, guessed transition locations have reached a state where one can expect that the movement of the stagnation points on all elements has stopped. This cycle is marked by the dotted line in Fig. 5 for the $\mathrm{Re}^{\text {lo }}$-take-off case. Thus, in the transition prediction iteration, the setting of $\Delta k_{c y c}=2,000$ provokes calls of the transition prediction module in a situation where the lift coefficient $c_{l}$ has not yet converged. However the convergence history of the lift coefficient is smooth and its evolution tendency is clear in these situations so that one can expect reasonable results from the transition prediction module anyhow. The dashed line marks 
the situation when the transition prediction module was called for the fourth and last time at cycle $\approx 10,350$. The continuing computation where the transition locations do not change anymore, is surprisingly - strongly perturbed and ends up with spiky oscillations in both the curves of the density residual and the force coefficients. The attempt to obtain a steady solution from an unsteady computation - marked by the long dashed line in Fig. 5 using a dual time stepping scheme ${ }^{10}$ also failed. In Fig. 6 , the convergence histories are shown for a computation which was started with a restart solution from cycle $\approx 8,850$. In this situation, the force coefficients have reached a state of convergence where their values changed only slightly during the last 850 cycles and the value of the density residual is sufficiently small. Thus, one can hope that the numerical disturbances which have remained

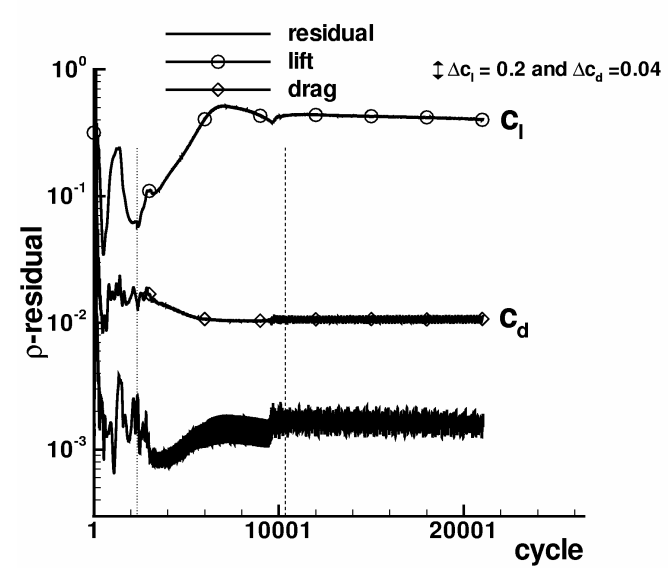

Fig. 7 Convergence history of the RANS computation, high Reynolds number takeoff case, dotted: transition prediction start, dashed: transition prediction end.

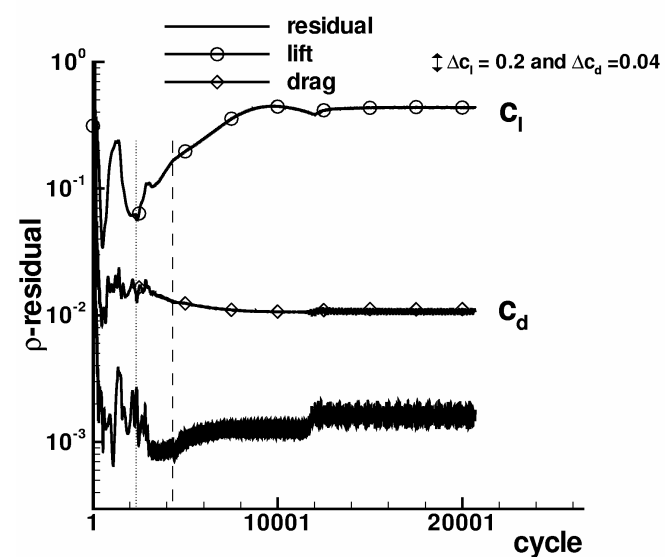

Fig. 8 Convergence history of the RANS computation, high Reynolds number takeoff case, dotted: here the transition prediction process had started, long dashed: here the restart run started using fixed predicted transition locations.

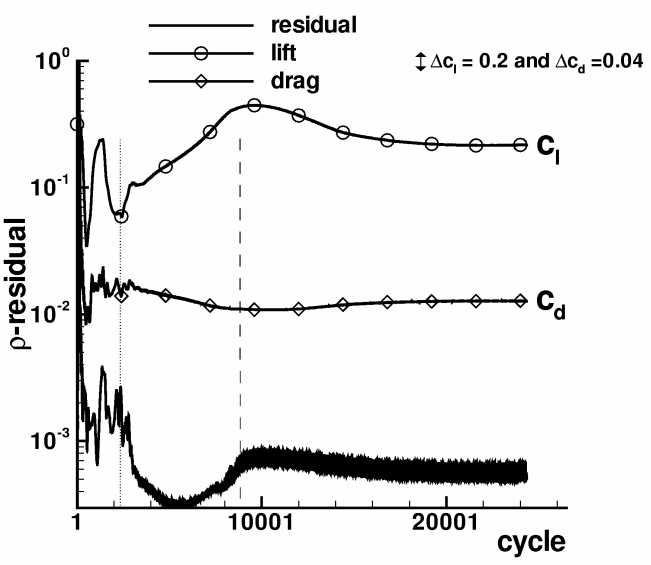

in the restart solution are well damped in the new computation which was performed using the transition lo-

Fig. 6 Convergence history of the RANS computation, low Reynolds number take-off case, dotted: here the transition prediction process had started, long dashed: here the restart run started using fixed predicted transition locations.

cations which were predicted by the transition location iteration before. Now, the convergence histories are very smooth, do not show any perturbations and end up in a steady solution based on a steady computation. Figs. 7 and 8 show a similar behavior of $\mathrm{Re}^{\mathrm{hi}}$-take-off case. In this case however, it was necessary to restart the computation based on a flow field solution from a very early convergence stage where the value of the density residual is sufficiently small, Fig. 8. Finally, the very smooth lift convergence curve reaches a steady state and the drag is oscillating around a steady average value. Why the computations produce a sudden rise of the density residual and the corresponding oscillations of the drag is not yet clear.

A close inspection of the computational grid showed, that the highest block-local density residuals were detected directly at the kinks of the blunt trailing edges. The grid points at these kinks belong to two different blocks with solid wall boundary condition. Very often, these points are the cause for convergence problems in a RANS computation when a cell-vertex spatial discretization scheme is used.

In Figs. 9-12, the convergence of the transition location iterations on the upper sides of the slat, the main airfoil and the flap for all test cases are depicted and the converged values are compared to experimental data as far as they are available. This is the case for the slat and the flap for all test cases ${ }^{22}$. In the figures the transition location values which come directly from the transition prediction module are depicted by dashed lines and the underrelaxed values by solid lines. A laminar separation point from the RANS computational grid which is used as a transition point is depicted by a square hollow symbol while a laminar separation point from the laminar boundary-layer code is depicted by a circular hollow symbol. A transition point due to TollmienSchlichting instabilities detected by the $\mathrm{e}^{N}$-database method is depicted by a black circular symbol. The values of the experimentally determined transition locations are marked by thin dashed lines. For the main airfoil, the location of the upper side 
kink is marked by a dashed-dotted line because transition is expected to occur in the vicinity of this location. The values of the transition points are normalized with respect to the chord length of the corresponding element, and are plotted against the RANS iteration cycles. Iterations start at cycle $=0$ which corresponds to the beginning of the computation, with transition prediction based on the restart solution from the computation with fixed, guessed transition locations. For the take-off cases, four steps of the transition location iteration are needed until the values of the transition points have converged. Due to the different convergence state of the restart solution, for the landing cases only three iteration steps are needed.
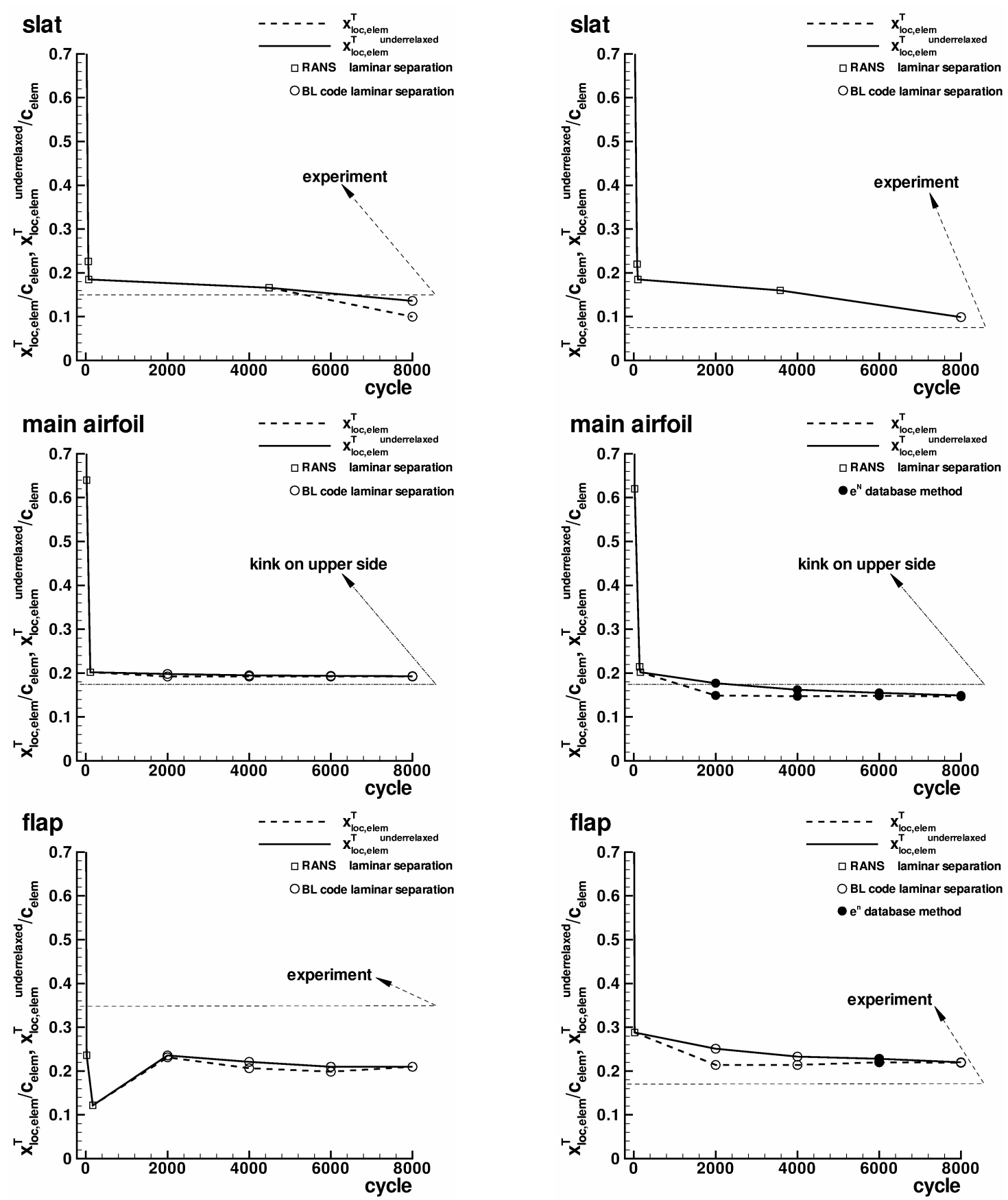

Fig. 9 Convergence histories of the transition Fig. 10 Convergence histories of the transition location iteration at the slat, main airfoil and flap, low Reynolds number take-off case. high Reynolds number take-off case. 

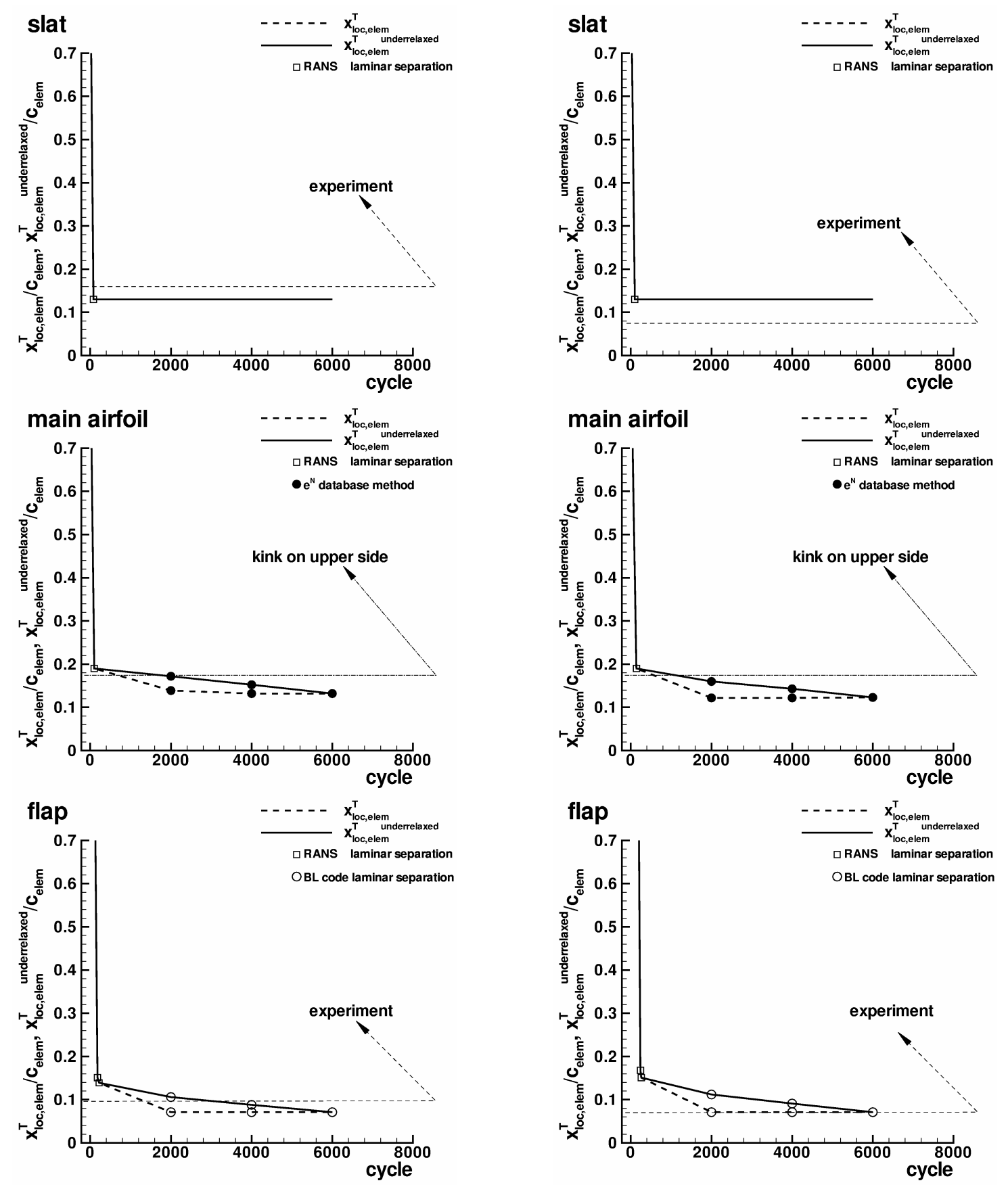

Fig. 11 Convergence histories of the transition Fig. 12 Convergence histories of the transition location iteration at the slat, main airfoil and flap, location iteration at the slat, main airfoil and flap, low Reynolds number landing case. high Reynolds number landing case.

At the $\mathrm{Re}^{\mathrm{lo}}$-take-off configuration, all transition points which occur in the transition prediction iteration are based on laminar separations during the transient phase of the computation, Fig. 9. All converged values come from the laminar boundary-layer method and yield an excellent approximation at the slat, a value in the area where transition is expected at the main airfoil, but a bad approximation at the flap where the predicted transition point is located too far upstream. For the upper side flap transition point, the approach of using the laminar separation point as an approximation of the real transition point fails when transition does not occur before the laminar boundary layer 
separates. A more capable transition prediction approach which is able to detect the transition point downstream of the point of laminar separation is needed here.

For the Re $e^{\text {hi }}$-take-off configuration, the converged values come from the laminar boundary-layer method at the slat and the flap and from the $\mathrm{e}^{N}$ database method at the main airfoil, Fig. 10, and yield a very good approximation at the slat, a value in the area where transition is expected at the main airfoil and a tolerable approximation at the flap. Here, it seems to be clear that transition did not occur due to Tollmien-Schlichting waves because one should have a value of the limiting $\mathrm{N}$-factor of $\left.N\right|_{x}{ }^{T} \approx 0.5$ to obtain the experimental value from the $\mathrm{e}^{N}$-database method. This value of the limiting $\mathrm{N}$-factor $\left.N\right|_{x^{T}}$ is definitely too small ${ }^{28,29}$. The application of criteria for bypass transition may yield a better approximation here.

Fig. 11 shows the results for the $\mathrm{Re}^{\mathrm{lo}}$-landing configuration. At the slat the final transition point comes from a laminar separation point detected in the RANS computational grid, instead from the laminar boundary-layer code which is normally the case. This is because usually, the prediction of laminar flow separation is more conservative in RANS methods compared to laminar flow separation prediction by boundary-layer methods, which indicate laminar separation at a position further upstream. At first sight this result is surprising, but it is an effect which is purely based on the details of the underrelaxation technique. The laminar separation point from the boundary-layer code in the last step of the transition location iteration is $x_{\text {sep,slat }} / \mathrm{c}_{\text {slat }} \approx 0.091$, but it is not taken into account by the underrelaxation algorithm due to the underrelaxation parameter settings for the slat. At the main airfoil, the converged value is due to Tollmien-Schlichting waves, and is located in the area where transition is expected. At the flap it is approximated by a laminar separation point from the boundary-layer code. The quality of the approximation at the slat and the flap is good.

Finally, the results of the $\mathrm{Re}^{\mathrm{hi}}$-landing configuration are shown in Fig. 12. At the slat the final transition point comes from a laminar separation point detected in the RANS computational grid, for the same reason as for the $\mathrm{Re}^{\mathrm{lo}}$-landing configuration. In contrast to the $\mathrm{Re}^{\mathrm{lo}}$-landing configuration where the final transition point is located a little bit too far upstream compared to the experimental value (which results in an approximation better than if the laminar separation point from the boundary-layer code had been used), the final transition point is located significantly too far downstream of the experimental value. Furthermore, the laminar separation point from the boundarylayer code which is located at $x_{\text {sep,slat }} / \mathrm{c}_{\text {slat }} \approx 0.097$ gives a very good approximation of the experimental value. This leads to the conclusion that for the fully automatic transition prediction procedure - a better approximation of the transition location on the slat is also possible by the manual input of the laminar separation point from the boundary layer code of course - the underrelaxation parameter settings must be very carefully adjusted on the one hand. On the other hand, it becomes evident that it is necessary to account for transition downstream of the point of laminar separation in order to get good results on the slat of the landing configuration in both cases. At the main airfoil, the converged value of the transition point is due to Tollmien-Schlichting waves and it is located in the area where transition is expected. At the flap it is approximated by a laminar separation point from the boundary-layer code. The quality of the approximation at the flap is almost perfect.

An overview of the computed values of the transition locations, $\left(x_{\mathrm{tr}} / \mathrm{c}\right)_{\mathrm{elem}}$, and the agreement with the experimental values according to

$$
\Delta x_{\text {trelem }}=\left(x_{\text {tr,elem }}^{\text {comp }}-x_{\text {trelem }}^{\text {exp }}\right) / \mathrm{c}_{\text {elem }}
$$

is shown in Table 1, where for the main airfoil the location of the transition point relative to the upper side kink is

Table 1 Computed values of the transition locations, $\left(x_{\mathrm{tr}} / \mathrm{c}\right)_{\text {elem, }}$ at slat, main airfoil and flap and agreement with the experimental values. For the main airfoil the transition location relative to the upper side kink is given.

\begin{tabular}{|c|c|c|c|c|c|c|c|c|}
\hline \multirow[b]{3}{*}{ elem } & \multicolumn{4}{|c|}{ take-off } & \multicolumn{4}{|c|}{ landing } \\
\hline & \multicolumn{2}{|c|}{$\operatorname{Re}^{\text {lo }}$} & \multicolumn{2}{|c|}{$\operatorname{Re}^{\text {hi }}$} & \multicolumn{2}{|c|}{$\operatorname{Re}^{\text {lo }}$} & \multicolumn{2}{|c|}{$\mathrm{Re}^{\mathrm{hi}}$} \\
\hline & $\left(x_{\mathrm{tr}} / \mathrm{c}\right)_{\mathrm{elem}}$ & $\Delta x_{\text {tr,elem }}$ & $\left(x_{\mathrm{tr}} / \mathrm{c}\right)_{\mathrm{elem}}$ & $\Delta x_{\text {tr,elem }}$ & $\left(x_{\mathrm{tr}} / \mathrm{c}\right)_{\text {elem }}$ & $\Delta x_{\text {tr,elem }}$ & $\left(x_{\mathrm{tr}} / \mathrm{c}\right)_{\text {elem }}$ & $\Delta x_{\text {tr,elem }}$ \\
\hline Slat & 0.136 & $-1.4 \%$ & 0.099 & $2.15 \%$ & 0.13 & $-3.04 \%$ & 0.13 & $5.3 \%$ \\
\hline Main & 0.193 & $1.46 \%$ & 0.149 & $-2.94 \%$ & 0.132 & $-4.64 \%$ & 0.123 & $-5.54 \%$ \\
\hline Flap & 0.2098 & $-13.7 \%$ & 0.22 & $4.86 \%$ & 0.071 & $-2.85 \%$ & 0.071 & $0.011 \%$ \\
\hline
\end{tabular}


given. In Table 2, the corresponding transition lengths, $\left(l_{\mathrm{tr}} / \mathrm{c}\right)_{\mathrm{elem}}$, which should be in the order of magnitude of about $5 \%$ of the chord length of the particular element, $l_{\mathrm{tr}, \mathrm{elem}}=5 \% \mathrm{c}_{\mathrm{elem}}$, are given.

Table 2 Computed values of the transition lengths, $\left(l_{\mathrm{tr}} / \mathrm{c}\right)_{\mathrm{elem}}$, at slat, main airfoil and flap.

\begin{tabular}{|c|c|c|c|c|}
\hline \multirow[b]{2}{*}{ elem } & \multicolumn{2}{|c|}{ take-off } & \multicolumn{2}{|c|}{ landing } \\
\hline & $\left(l_{\mathrm{tr}} / \mathrm{c}\right)_{\text {elem }}$ for $\mathrm{Re}^{\mathrm{lo}}$ & $\left(l_{\mathrm{tr}} / \mathrm{c}\right)_{\text {elem }}$ for $\mathrm{Re}^{\mathrm{hi}}$ & $\overline{\left(l_{\mathrm{tr}} / \mathrm{c}\right)_{\text {elem }} \text { for } \mathrm{Re}^{\mathrm{lo}}}$ & $\left(l_{\mathrm{tr}} / \mathrm{c}\right)_{\text {elem }}$ for $\mathrm{Re}^{\mathrm{hi}}$ \\
\hline Slat & $6.76 \%$ & $4.75 \%$ & $6.94 \%$ & $5.37 \%$ \\
\hline Main & $3.57 \%$ & $5.08 \%$ & $7.01 \%$ & $5.35 \%$ \\
\hline Flap & $4.87 \%$ & $3.96 \%$ & $3.16 \%$ & $2.7 \%$ \\
\hline
\end{tabular}

In Figs. 13-16, the $c_{\mathrm{p}^{-}}$and $c_{\mathrm{f}}$-distributions of the four cases are shown. The $c_{\mathrm{p}}$-distribution of the Re $\mathrm{R}^{\mathrm{lo}}$-take-off
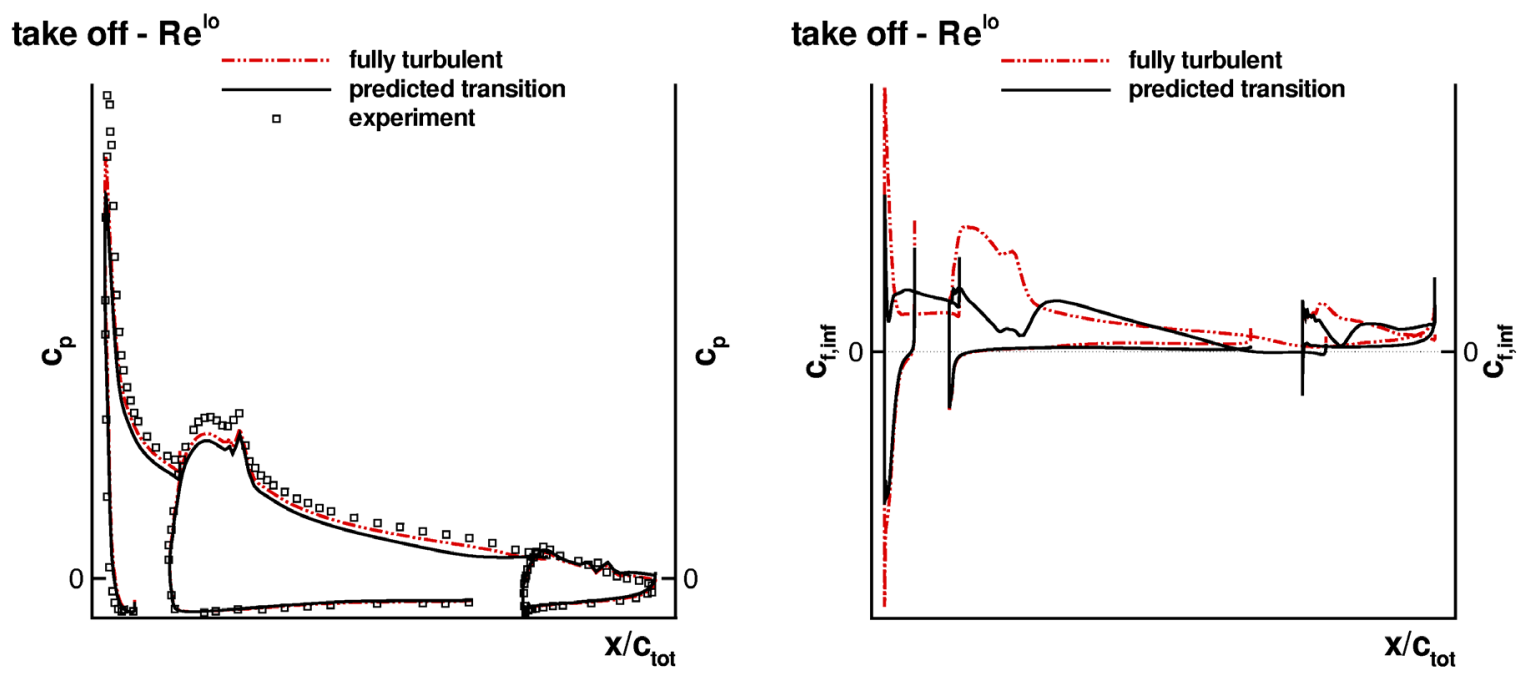

Fig. $13 c_{\mathrm{p}}$ - and $c_{\mathrm{f}}$-distributions, low Reynolds number take-off case, SAE turbulence model.
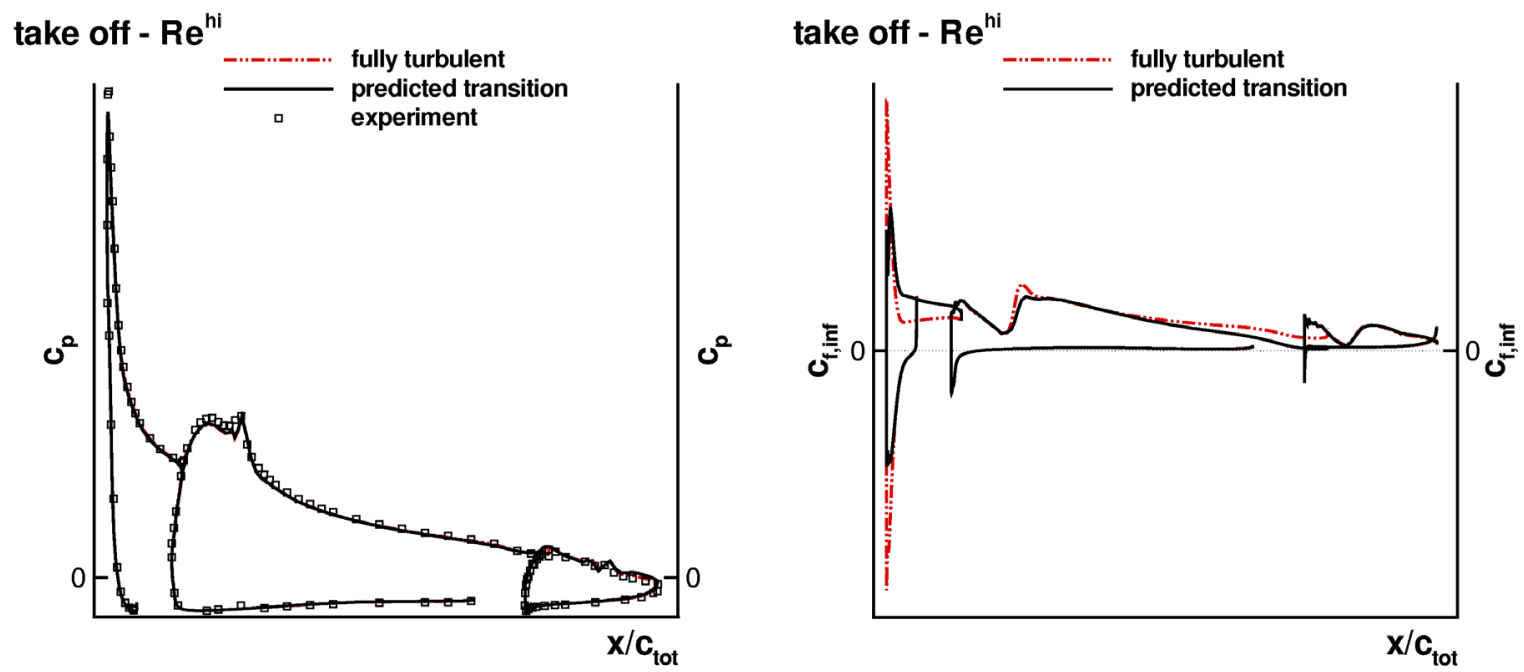

Fig. $14 c_{\mathrm{p}}$ - and $c_{\mathrm{f}}$-distributions, high Reynolds number take-off case, SAE turbulence model.

case, Fig. 13, quantitatively does not agree well with the experimental one on the slat and the main airfoil, and even exhibits higher deviations than the $c_{\mathrm{p}}$-distribution of a fully turbulent computation, especially in the upper side 
suction peak areas. This is an unexpected result and can be explained with the existence of the trailing edge separation bubble at the main airfoil upper side which does not appear in the fully turbulent computation. For the $\mathrm{Re}^{\text {hi }}$-take-off case, Fig. 14, the agreement of experimental and computed $c_{\mathrm{p}}$-distributions is much better. Although also in this case the suction peak at the slat is not reached, the overall agreement is satisfying. The $c_{\mathrm{p}}$-distributions of the fully turbulent computation and the computation with transition are almost the same, and even the $c_{\mathrm{f}}$-distributions of both computations match in large portions of the main airfoil and the flap. On the upper sides of both components, the minimum $c_{\mathrm{f}}$-values in the transitional flow regions are the same and they are located at the same positions. The $c_{\mathrm{f}}$-rise in the transition region of the main airfoil is more abrupt in the fully turbulent case than in the case with transition locations and transition lengths, and at the flap the computed $c_{\mathrm{f}}$-distributions are almost identical. In this high Reynolds number case, the turbulence model used is able to simulate laminar flow and transition to turbulence without having prescribed laminar flow regions in the computational grid, for two of the three components of the configuration. This behaviour may be due to the high Reynolds number (and the corresponding low value of the molecular viscosity), which has a damping influence on the turbulence production term of the SAE model. The results of the computation with transition show that on the main airfoil upper side, the flow is very near to separation at the trailing edge in contrast to the fully turbulent results.

Fig. 15 shows the results for the $\mathrm{Re}^{\mathrm{lo}}$-landing case. Here, a clear improvement with respect to the predicted
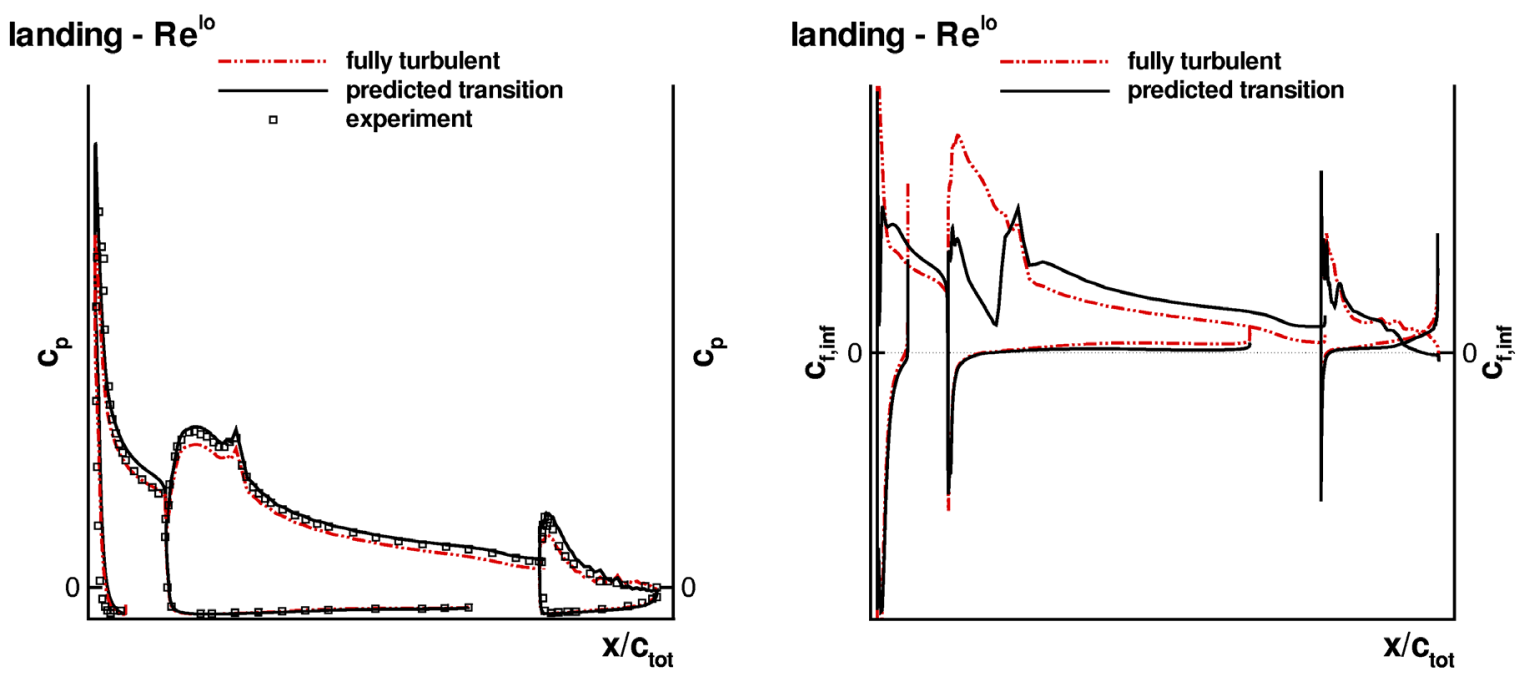

Fig. $15 c_{\mathrm{p}}$ - and $c_{\mathrm{f}}$-distributions, low Reynolds number landing case, Wilcox k- $\omega$ turbulence model.
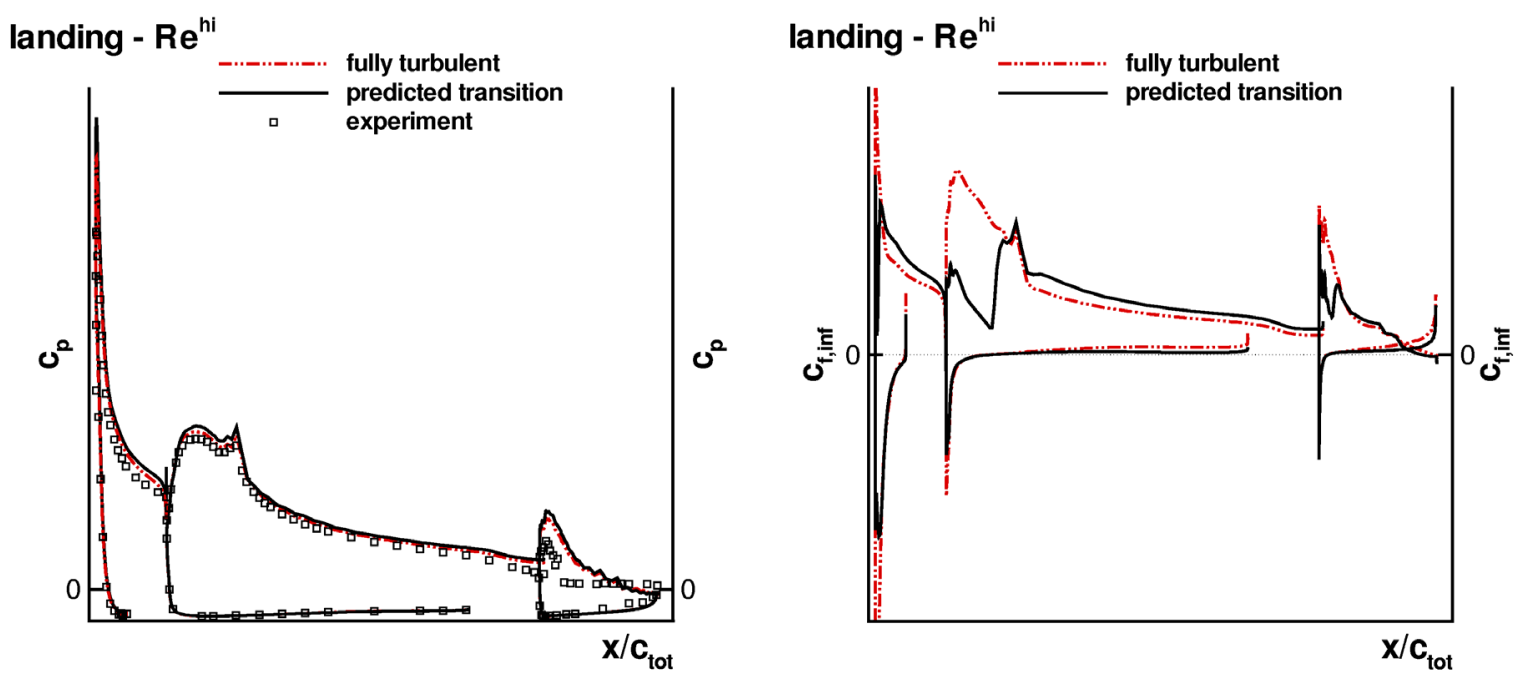

Fig. $16 c_{\mathrm{p}^{-}}$and $c_{\mathrm{f}}$-distributions, high Reynolds number landing case, Wilcox k- $\omega$ turbulence model. 
$c_{\mathrm{p}}$-distributions on the main airfoil and in the suction area of the flap is obtained in the computation with transition. The suction peak on the slat is overestimated in the computation with transition, whereas in the fully turbulent computation the suction peak is not reached. The flap separation bubble in the computation with transition leads to a slight underestimation of the pressure level at the flap trailing edge. At the main airfoil, the turbulent skin friction level is significantly higher in the computation with transition than in the fully turbulent computation. Finally, in Fig. 16, the results for the Re ${ }^{\text {hi }}$-landing case are depicted. Here both, the computation with transition and the fully turbulent computation clearly overestimate the suction peak at the slat, and in both cases the large separation bubble on the flap upper side which is clearly visible in the experimental $c_{\mathrm{p}}$-distribution is not predicted. This leads to an overestimation of the overall $c_{\mathrm{p}}$-level on all elements. The differences between the results from the computation with transition and the fully turbulent computation are as expected for the $c_{\mathrm{p}}$-distributions as well as for the $c_{\mathrm{f}}$-distributions.

Because the computational results for the take-off configuration using the SAE turbulence model were not of sufficient quality with respect to the accuracy of the predicted pressure distributions, the computations were repeated applying the Wilcox k- $\omega$ turbulence model using the transition locations and transition lengths determined in the SAE computations. The computational results are shown in the Figs. 17 and 18. Now, for both Reynolds numbers, the suction peaks at the slat, and for the low Reynolds number the experimental pressure level on the slat upper side, are reached with very good accuracy. The pressure levels on the main airfoil upper side are improved visibly in both
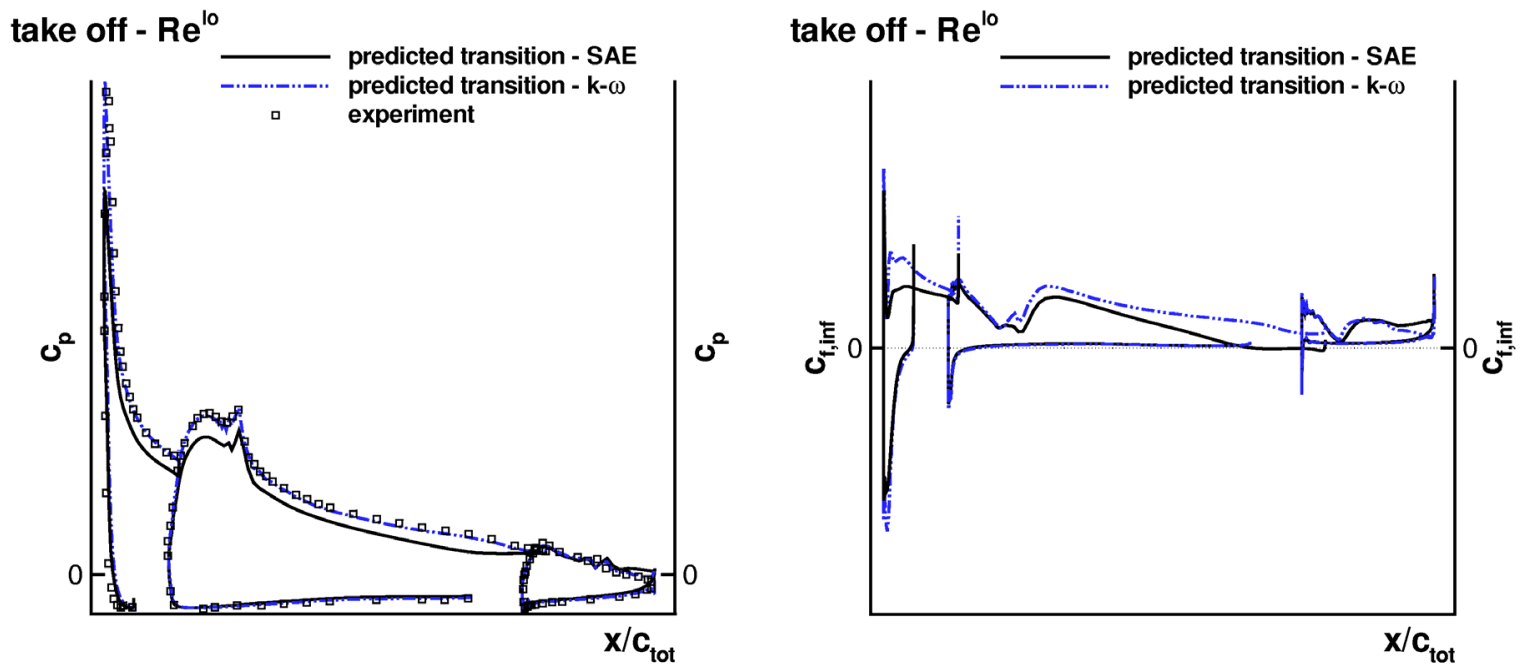

Fig. $17 c_{\mathrm{p}^{-}}$and $c_{\mathrm{f}}$-distributions, low Reynolds number take-off case, Wilcox k- $\omega$ turbulence model.
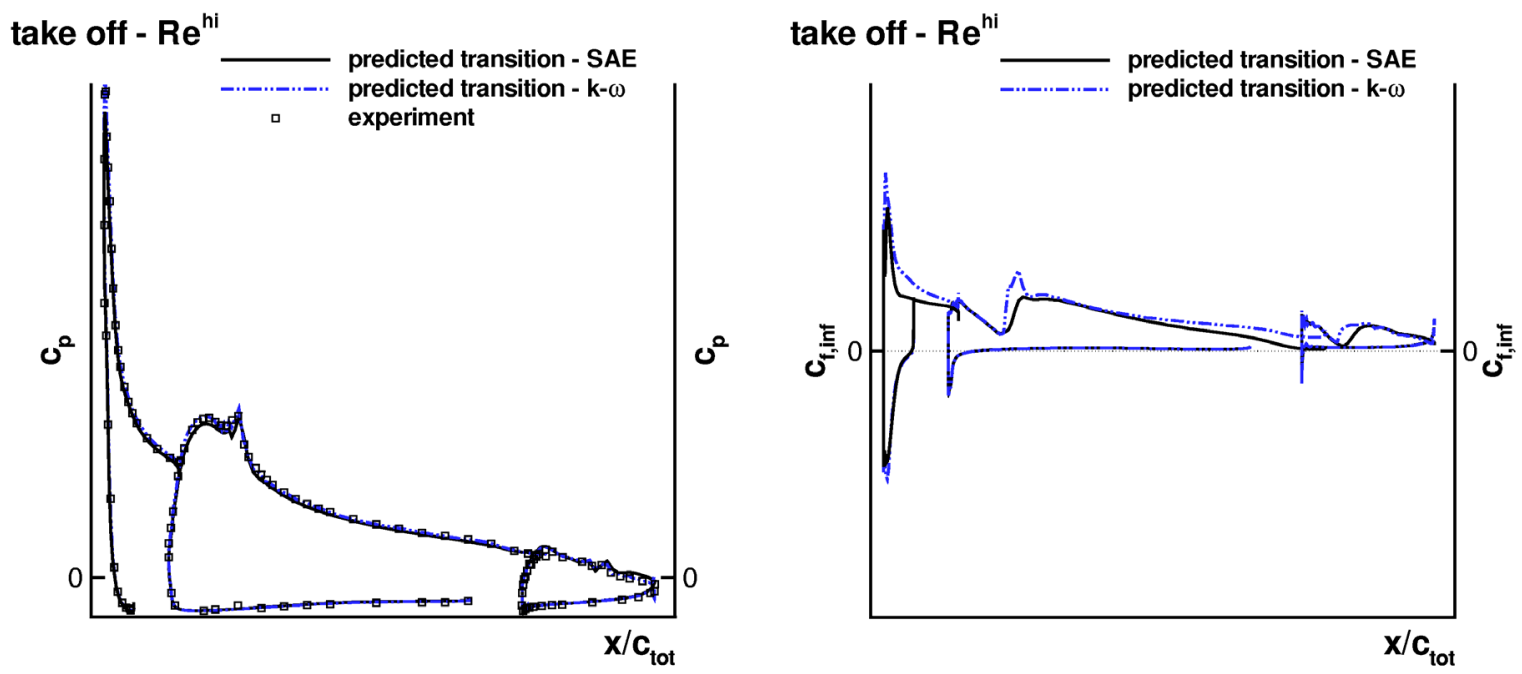

Fig. $18 c_{p^{-}}$and $c_{\mathrm{f}}$-distributions, high Reynolds number take-off case, Wilcox k- $\omega$ turbulence model. 
cases. The improvement for the low Reynolds number case is significant. The deviations between the experimental and the computed $c_{\mathrm{p}}$-distributions at the flap almost do not appear anymore. Remarkable are the differences in the $c_{\mathrm{f}}$-distributions, especially in the transition regions. In both computations using the Wilcox k- $\omega$ model, the $c_{\mathrm{f}}$-rise directly downstream of the transition point occurs more rapidly and more intensely for all three elements than in the computations using the SAE model, and the local maximum $c_{\mathrm{f}}$-value at the beginning of the fully turbulent flow region is greater. In both cases, the turbulent boundary layer at the upper side trailing edge of the main airfoil is far from separation with the Wilcox $\mathrm{k}-\omega$ model.

To verify if the unsatisfactory results using the SAE model originate from the application of the transition lengths and the intermittency function, the computations with the SAE model were repeated using the same transition locations but applying point transition instead of the transitional flow models. In both cases, the $c_{\mathrm{p}}$-distributions are almost the same as the ones with transitional flow models. The $c_{\mathrm{f}}$-distributions for the $\mathrm{Re}^{\mathrm{lo}}$-take-off case are almost the same as for the case with transitional flow models at the slat and the main airfoil. At the flap the $c_{\mathrm{f}}$-rise directly downstream of the transition point starts a bit sooner that is the case with transitional flow models. For the Re ${ }^{\text {hi }}$-take-off case, at the slat and the flap the $c_{\mathrm{f}}$-distributions are almost identical to the ones from the computations with transitional flow models. At the main airfoil the $c_{\mathrm{f}}$-distribution is identical to the one from the fully turbulent computation.

It was also tested if the value of the turbulence production limiter $C_{P}$ has an effect on the solution. Using $C_{P}=10$ and the SAE model for $\mathrm{Re}^{\mathrm{lo}}$-take-off case led to divergence of the computation restarted with the converged solution obtained before with $C_{P}=10^{10}$. Using $C_{P}=10^{10}$ and the Wilcox k- $\omega$ model for Re ${ }^{\text {lo }}$-take-off case yielded a visible attenuation of the oscillations which had remained in the converged solution, but the steady average values of the lift and drag coefficients did not change. The $c_{\mathrm{p}}$ - and $c_{\mathrm{f}}$-distributions changed very slightly in the regions of the trailing edges. The transitional flow regions were almost not affected. This outcome indicates that the properties of the turbulence model itself are the cause for the unexpected behavior of the SAE model in the computations with predicted transition locations.

Finally, an overview over the experimentally measured and computed lift coefficients, $c_{1}$, and drag coefficients, $c_{\mathrm{d}}$, is presented in Figs. 19 and 20, where the experimental drag coefficient values were determined by far wake surveys $^{22}$ - carried out perpendicularly to the free stream velocity - including all uncertainties which are known for far wake drag estimates performed on high-lift configurations. Usually the far wake drag estimates are done based on total pressures which do not account for the very high angle of the flow downwash so that low estimates of drag values are a consequence.

Fig. 19 shows this comparison for the take-off configuration, which exhibits a trend of the experimental lift coefficient to lower values with increasing Reynolds number. This may be due to the separation bubble at the slat which was detected for the low Reynolds number, but did not occur for the high Reynolds number ${ }^{22}$. A closer inspection of the $c_{\mathrm{p}}$-distributions at the slat reveals the reported difference and visibly higher suction levels over wide portions of the slat upper side for the low Reynolds number. The very unsatisfactory computed $c_{1}$ values using

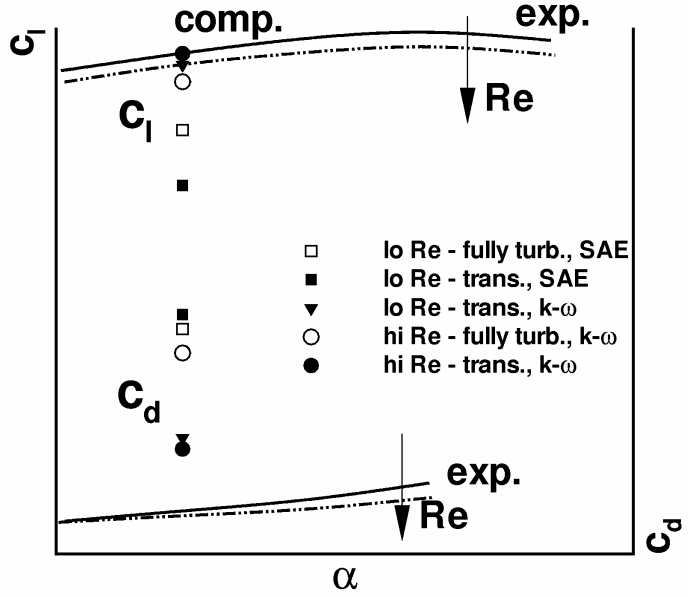

Fig. 19 Measured and computed lift coefficients, $c_{\mathrm{l}}$, and drag coefficients, $c_{\mathrm{d}}$, versus the angle of attack $\alpha$, take-off case.

the SAE turbulence model can be improved drastically, by using the Wilcox $\mathrm{k}-\omega$ model and the predicted transition locations, to results which differ from the experimental ones less then $1.5 \%$, that is $\Delta c_{1}<1.5 \%$, according to

$$
\Delta c_{\mathrm{k}}=\left(c_{\mathrm{k}}^{\text {comp }}-c_{\mathrm{k}}^{\exp }\right) / c_{\mathrm{k}}^{\exp }, \quad \mathrm{k}=1, \mathrm{~d} \text {. }
$$

The Reynolds number effect for the computed lift coefficients is contrary to the experimental trend, which is understandable because in the computations no separations bubbles are predicted. Thus one gets the usual Reynolds number effect, with higher suction levels on the slat upper side for the higher Reynolds number. The computed drag coefficients which differ significantly from the experimental far wake drag estimates, can be improved drastically as well when the predicted transition locations are applied and the Wilcox k- $\omega$ model is used. The differences between 
the experimental and the computed drag, $\Delta c_{\mathrm{d}}$, according to Eq. (23), decrease from over $120 \%$ for the computations with the SAE model to about 50\% using the Wilcox k- $\omega$ model. The Reynolds number effect in the computations is as it was found in the measurements. The possibility that the computed values of the drag coefficient of a high-lift configuration may deviate strongly from the experimental values is well known and reported for example in Ref. 30 where another configuration was investigated. There the comparison of the computed pressure drag with pressure drag estimates from the integrated experimental pressures, yielded good results in some cases. Unfortunately, the experimental pressure drags for the configurations investigated in this work are not available. Besides the uncertainties of the experimental approach of drag estimation, which definitely contributes to the difference between the experimental and the computed drag values, the differences between the computed and the measured pressures may be a cause of the deviations. In Ref. 31, this issue was investigated in detail and it was shown that the overall pressure drag of a high-lift configuration, which dominates the drag value of the configuration as a whole as well as the drag of every single element, is composed of a balance of very large positive and negative contributions. The contribution of one single element may be one order of magnitude larger than the resulting overall drag of the complete configuration. Thus, a relative error of $5 \%$ of the computed drag on the slat upper side may result in a change of $50 \%$ for the overall drag value ${ }^{31}$. At this point, as so often, the question arises: how much the transition prediction and transition modeling on the one hand, and the turbulence modeling on the other hand, are responsible for this effect and how much they contribute to it.

For the landing configuration measurements, random occurrence of flow separation over the flap is reported for the low Reynolds number. Also for the high Reynolds number, separation over the flap was observed, but here it was possible to reduce this phenomenon by cleaning the wind tunnel model.

The experimental force coefficient values for the $\mathrm{Re}^{\text {lo }}$-landing case in Fig. 20 do not correspond to the experimental $c_{\mathrm{p}}$-distribution in Fig. 15 with attached flow over the flap, but to the separated flow case whose $c_{\mathrm{p}}$-distribution is not shown in this presentation. For the $\mathrm{Re}^{\text {hi }}$-landing case, the experimental force coefficient values in Fig. 20 do not correspond to the experimental $c_{\mathrm{p}}$-distribution in Fig. 16 with separated flow over the flap,

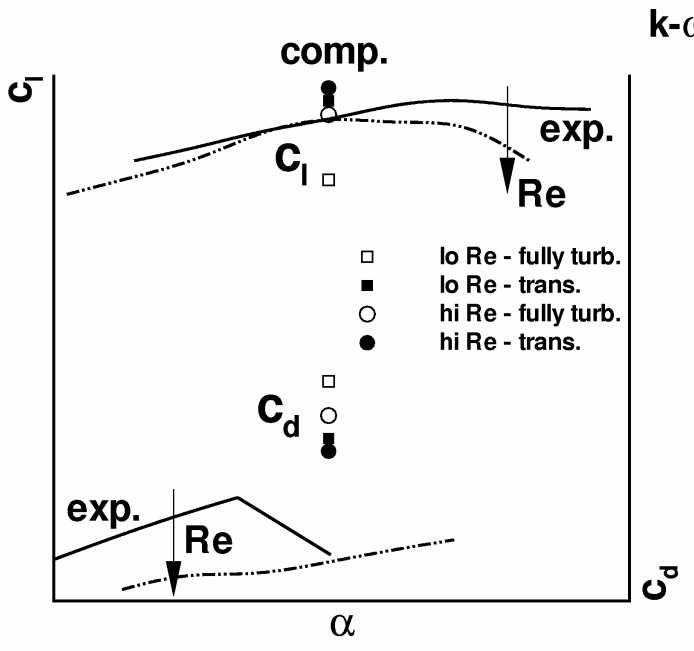

Fig. 20 Measured and computed lift coefficients, $c_{\mathrm{l}}$, and drag coefficients, $c_{\mathrm{d}}$, versus the angle of attack $\alpha$, landing case.

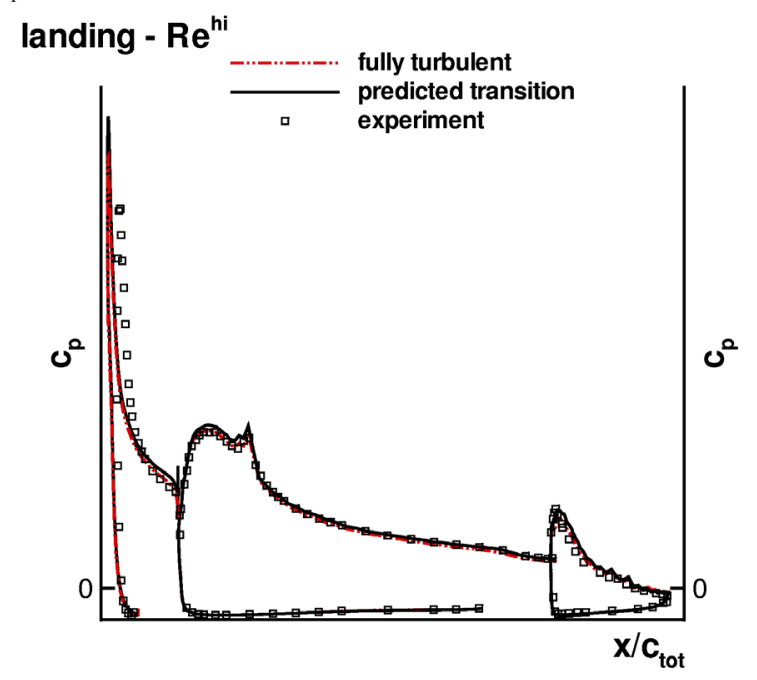

Fig. $21 c_{\mathrm{p}}$-distributions, high Reynolds number landing case, Wilcox $k-\omega$ turbulence model, experimental distribution taken at the cleaned wind tunnel model with attached flow over the flap. but to the case with attached flow over the flap of the cleaned wind tunnel model. The experimental $c_{\mathrm{p}}$-distribution for this flow case is compared to the one computed in Fig. 21. In this comparison, one finds a significantly improved matching of the experimental and computed $c_{\mathrm{p}}$-distributions on the flap and also on the main airfoil. At the slat however, the suction peak still seems to be highly overestimated. Moreover, the deviation of the location of the suction peak has increased visibly in this comparison, which would mean a worsening of the experimental and computed agreement, compared to the flow case with flow separation over the flap.

The Reynolds number trend of the experimental lift coefficient is similar to the take-off configuration, also the same differences with respect to the existence of the slat separation bubble are reported. ${ }^{22}$ These are visible in a close inspection of the corresponding $c_{\mathrm{p}}$-distributions, and the same circumstance in the computations - no separation on the slat - as in the take-off cases occurs. For the $\mathrm{Re}^{\mathrm{lo}}$-landing case, the computation with transition reduces the error in the computed lift coefficient from over $9 \%$ to less than $3 \%$. For the $\mathrm{Re}^{\mathrm{hi}}$-landing case, the $c_{\mathrm{l}}$-value of the 
fully turbulent computation has an error of less than $1 \%$, while the value of the computation with transition has an error of almost 5\%. In the computations, one gets the usual Reynolds number effect, with higher lift for a higher Reynolds number.

The differences between the experimental and the computed drag, $\Delta c_{\mathrm{d}}$, according to Eq. (23) decrease from about $98 \%$ for the fully turbulent computations of both cases, to about $66 \%$ for the computations of both cases with transition. The Reynolds number effect in the computations is as it was found in the measurements.

Finally it must be emphasised here, that the wake measurements at the landing configuration were affected by the occurrence of the separation over the flap, ${ }^{22}$ which led to an unsteady flow in these situations. This brings up the question of how much of the unsteadiness may have remained in the reported values of the force coefficients.

\section{Conclusion}

The DLR RANS solver FLOWer was coupled to the DLR transition prediction module in order to perform RANS computations of high-lift multi-element systems with automatic laminar-turbulent transition prediction. It could be shown, that the coupled system represents a RANS-based CFD tool that provides accurate values of the transition locations during the ongoing RANS computation automatically and fast without the need for the intervention by the code user. Thus, RANS computations of high-lift multi-element configurations with transition can be carried out without a priori knowledge of the transition characteristics of the specific flow problem.

The transition prediction coupling procedure was successfully applied to a number of 3-element high-lift test cases. The transition location iterations converge fast and the predicted transition locations are partly of excellent accuracy. Nevertheless, in some cases the accuracy must be improved by taking into account other transition prediction approaches which are able to detect the transition point downstream of the point of laminar separation, or which detect bypass transition. All transition lengths are of the expected order of magnitude.

The comparison of the computed and the experimentally determined pressure distributions yields a good agreement for the two take-off test cases and a satisfying agreement for the two landing cases when the Wilcox k- $\omega$ turbulence model was used. The computed lift coefficients agree well with the experimental findings. However, the computed drag coefficients, show significant deviations from the experimental values. In this respect, the abilities of the turbulence models used must be questioned, and other turbulence models must be tested. Nevertheless, the results of the computations with predicted transition locations yield a significantly lower error in the computed drag values - the error decreases by about $30 \%$ - compared to the results from fully turbulent computations.

The turbulence model used may have - although it almost does not influence the results of the transition prediction procedure - a significant impact on the computed $c_{\mathrm{p}}$ - and $c_{\mathrm{f}}$-distributions and on the force coefficients. The use of the same values of the transition locations and the transition lengths led to the expected improvement of the computed quantities compared to the results from fully turbulent computations in all cases when the Wilcox k- $\omega$ turbulence model was used. For one of the two cases which were computed using the Spalart-Allmaras model with Edwards and Chandra modification however, the results from the computation with transition exhibited significantly larger deviations from the experimental data than the fully turbulent computational results.

Finally, the application of more capable transition prediction approaches and other transition criteria must be tested and their impact on the pressure distribution and the force coefficients must be investigated. The consideration of these transition prediction issues will be addressed within the European project EUROLIFT II ${ }^{32}$.

\section{Acknowledgments}

This work has partly been carried out within the HiAer Project (High Level Modelling of High Lift Aerodynamics) ${ }^{33}$. The HiAer project is a collaboration between DLR, ONERA, KTH, HUT, TUB, Alenia, Airbus Deutschland, QinetiQ and FOI. The project is managed by FOI and is partly funded by the European Union (Project Ref: G4RDCT-2001-00448).

We acknowledge BAE Systems for letting us use the 59\% section data of a representative civil aircraft wing in this study.

\section{References}

${ }^{1}$ Radespiel, R., Graage, K., Brodersen, O., "Transition Predictions Using Reynolds-Averaged Navier-Stokes and Linear Stability Analysis Methods“, AIAA Paper 91-1641, 1991

${ }^{2}$ Smith, A.M.O., Gamberoni, N., "Transition, Pressure Gradient and Stability Theory“, Douglas Aircraft Company, Long Beach, Calif. Rep. ES 26388, 1956

${ }^{3}$ van Ingen, J.L., "A suggested Semi-Empirical Method for the Calculation of the Boundary Layer Transition Region", University of Delft, Dept. of Aerospace Engineering, Delft, The Netherlands, Rep. VTH-74, 1956 
${ }^{4}$ Stock, H. W., Haase, W., "A Feasibility Study of $\mathrm{e}^{N}$ Transition Prediction in Navier-Stokes Methods for Airfoils", AIAA Journal, Vol.37, no. 10, 1999, pp. 1187-1196

${ }^{5}$ Horton, H. P., Stock, H. W., "Computation of Compressible, Laminar Boundary Layers on Swept, Tapered Wings“, Journal of Aircraft, Vol.32, 1995, pp. 1402-1405

${ }^{6}$ Stock, H. W., Degenhardt, E., "A simplified e ${ }^{N}$ method for transition prediction in two-dimensional, incompressible boundary layers", Zeitung für Flugwissenschaft und Weltraumforschung, Vol.13, 1989, pp. 16-30

${ }^{7}$ Warren, E. S., Hassan, H. A., "Transition Closure Model for Predicting Transition Onset", Journal of Aircraft, Vol.35, 1998, pp. $769-775$

${ }^{8}$ Czerwiec, R.M., Edwards, J. R., Rumsey, C. L., Bertelrud, A., Hassan, H. A., "Study of High-Lift Configurations Using k- $\zeta$ Transition/Turbulence Model“, AIAA Paper 99-3186, 1999

${ }^{9}$ Edwards, J. R., Roy, C. J., Blottner, F. G., Hassan, H. A., "Development of a One-Equation Transition/Turbulence Model“, AIAA Journal, Vol.39, no. 9, 2001, pp. 1691-1698

${ }^{10}$ FLOWer - Installation and User Handbook, Release 116, Doc.Nr. MEGAFLOW-1001, Institut für Entwurfsaerodynamik, Deutsches Zentrum für Luft- und Raumfahrt e.V., 2000

${ }^{11}$ Krumbein, A., Stock, H. W., "Laminar-turbulent Transition Modeling in Navier-Stokes Solvers using Engineering Methods“, ECCOMAS 2000, Barcelona (e), 11.-14. September 2000, ECCOMAS 2000 - CD-Rom Proceedings, editor: International Center for Numerical Methods in Engineering (CIMNE), 2000, ISBN: 84-89925-70-4, Depósito Legal: B-37139-2000

${ }^{12}$ Krumbein, A., "Coupling of the DLR Navier-Stokes Solver FLOWer with an $\mathrm{e}^{N}$-Database Method for laminar-turbulent Transition Prediction on Airfoils", New Results in Numerical and Experimental Fluid Mechanics III, Notes on Numerical Fluid Mechanics - Vol.77, Berlin, Heidelberg, New York, Springer Verlag, 2002, pp. $92-99$

${ }^{13}$ Stock, H. W., Haase, W., "Navier-Stokes Airfoil Computations with $\mathrm{e}^{N}$ Transition Prediction Including Transitional Flow Regions", AIAA Journal, Vol.38, no. 11, 2000, pp. 2059-2066

${ }^{14}$ Krumbein, A., "Transitional Flow Modeling and Application to High-Lift Multi-Element Airfoil Configurations“,Journal of Aircraft, Vol.40, 2003, pp. 786-794

${ }^{15}$ Dhawan, S., Narasimha, R., "Some properties of boundary layer flow during the transition from laminar to turbulent motion“, Journal of Fluid Mechanics, Vol.3, 1958, pp. 418-436

${ }^{16}$ Walker, G. J., "Transitional Flow on Axial Turbomachine Blading“, AIAA Journal, Vol.27, No. 5, 1989, pp. 595- 602

${ }^{17}$ Stock, H. W., Haase, W., "Some Aspects of Linear Stability Calculations in Industrial Applications", Transitional Boundary Layers in Aeronautics, editors: Henkes, R. A. W. M.; van Ingen, J.L., 1996, pp. 225-238

${ }^{18}$ Stock, H. W., "Navier-Stokes Computations of Laminar Airfoils Using e ${ }^{N}$ Transition Prediction",German Aerospace Center - DLR, Braunschweig, Germany, Internal Report IB 129-99/18, 1999

${ }^{19}$ Dargel, G., Schnieder, H., "GARTEUR AD (AG08) Final Report“, GARTEUR High Lift Action Group AD (AG08), TP043, MBB Transport- und Verkehrsflugzeuge, Bremen, 1989

${ }^{20}$ Thibert, J. J., "The GARTEUR High Lift Research Programme“, AGARD Conference Proceedings 515 - High-Lift System Aerodynamics, 1993, pp. 16-1 - 16-21

${ }^{21}$ Brodersen, O., Ronzheimer, A., Ziegler, R., Kunert, T., Wild, J., Hepperle, M., “Aerodynamic Applications using MegaCads“, Proc. of 6th International Conference on Numerical Grid Generation in Computational Field Simulation, editor: M. Cross, publisher: ISGG, NSF Eng. Research Center, Mississippi State University, 1998, pp. 793-802

${ }^{22}$ Manie, F., Piccin, O., Ray, J.P., "Test Report of the 2D Model M1 in the ONERA F1 Wind Tunnel”, GARTEUR AD (AG08), TP-041, ONERA, Chatillon, October 1989

${ }^{23}$ Spalart, P. R., Allmaras, S. R., “A one-equation turbulence model for aerodynamic flows", La Recherche Aérospatiale, $1994, \mathrm{n}^{\circ} 1$, pp. $5-21$

${ }^{24}$ Edwards, J.R., Chandra, S., "Comparison of Eddy Viscosity - Transport Turbulence Models for Three-Dimensional, ShockSeparated Flowfields", AIAA Journal, Vol.34, No. 4, 1996, pp. 756-763

${ }^{25}$ Wilcox, D. C., "Reassessment of the Scale-Determining Equation for Advanced Turbulence Models," AIAA Journal, Vol. 26, No. 11, 1988, pp. 1299-1310

${ }^{26}$ Stock, H. W., Krumbein, A., HiAer Deliverable D1.3-2: "2D High-Lift RANS Computations with e $\mathrm{N}^{\mathrm{N}}$ Transition Prediction - A310 Take-Off and Landing Configurations“, Technical Report, DLR, Braunschweig, April 2003

${ }^{27}$ High Level Modelling of High Lift Aerodynamics-HiAer, "Final Technical Report", GROWTH Project GRD1-2000-25226 HiAer, April 2004

${ }^{28}$ Arthur, M. T., Dol, H., Krumbein, A., Houdeville, R., Ponsin, J., "Application of Transition Criteria in Navier-Stokes Computations", GARTEUR AD (AG-35), TP-137, ONERA, Toulouse, January 2003

${ }^{29}$ Stock, H. W., "Infinite Swept Wing RANS Computations with $\mathrm{e}^{N}$ Transition Prediction, a Validation Study", ALTTA Report D3.1.2-4, October 2002, submitted to AIAA Journal, August 2002

${ }^{30}$ K.M.J. de Cock, "2D maximum lift prediction for the 59 percent span wing section of the A310 aircraft", NLR-CR-98500, NLR, Amsterdam, November 1998

${ }^{31}$ Rudnik, R., Ronzheimer, A., Schenk, M., „Berechnung von zwei- und dreidimensionalen Hochauftriebskonfigurationen durch Lösung der Navier-Stokes Gleichungen“, DGLR-Jahrbuch 1996 - Bd. II, JT 96-104, 1996, pp. 717-726,

${ }^{32}$ EUROLIFT II-European High Lift Programme II, Annex B: "Description of Work”, A Specific Targeted Research Project of the $6^{\text {th }}$ European Framework Program, GRD-2004-502896, 2004

${ }^{33}$ High Level Modelling of High Lift Aerodynamics-HiAer, Annex 1, "Description of Work", GROWTH Project GRD12000-25226 HiAer, 2000 